Pacific Journal of Mathematics

COUNTEREXAMPLE TO A CONJECTURE OF H. HOPE 


\title{
COUNTEREXAMPLE TO A CONJECTURE OF H. HOPF
}

\author{
Henry C. Wente
}

The purpose of this paper is to produce an immersion of a compact oriented two-dimensional surface of genus one into Euclidean 3-space with constant mean curvature $H \neq 0$. We thus provide a counterexample in dimension 3 to the following conjecture of $\mathbf{H}$. Hopf.

Conjecture of $H$. Hopf. Let $\Sigma$ be an immersion of an oriented, closed hypersurface with constant mean curvature $H \neq 0$ in $R^{n}$. Must $\Sigma$ be the standard embedded $(n-1)$-sphere?

Two important results relating to this conjecture are due to A. D. Alexandrov and H. Hopf. A. D. Alexandrov [1] showed that the conjecture is true if $\Sigma$ is an embedded hypersurface in $R^{n}$. This extended an old result of J. H. Jellett [10] (see also [15] p. 354), who showed the conjecture to be valid in the case where $\Sigma$ is a two-dimensional star-shaped surface in $R^{3}$. H. Hopf himself [8] showed the conjecture to be true when $\Sigma$ is an immersion of $S^{2}$ into $R^{3}$ with constant mean curvature.

A negative answer to the Hopf conjecture in dimensions greater than three was recently supplied by Wu-Yi Hsiang [9]. He constructed a counterexample in $R^{4}$. He considered 3-dimensional immersions into $R^{4}$ which were invariant under the action of $O(2) \times O(2)$, a subgroup of the isometry group for $R^{4}$. If one identifies $R^{4}$ with $C \times C$ so that a point in $R^{4}$ has coordinates $\left(z_{1}, z_{2}\right)$ where $z_{i}=x_{i}+i y_{l}$ and the action of $O(2) \times$ $O(2)$ to be given by $\left(z_{1}, z_{2}\right) \rightarrow\left(e^{i \theta} z_{1}, e^{i \alpha} z_{2}\right)$, then the orbit space is $R^{4} / O(2) \times O(2)=\left\{\left(x_{1}, x_{2}\right) \mid x_{1} \geq 0, x_{2} \geq 0\right\}$ and a surface of constant mean curvature with the desired symmetry is determined by a generating curve lying in the orbit space. Such a curve will generate a closed surface if it terminates on the positive $x_{1}$ and $x_{2}$ axes. Hsiang succeeded in showing that there exist such curves which generate an immersion of $S^{3}$ into $R^{4}$ of constant mean curvature which is not a standard sphere. This method does not carry over to the classical dimension and so the Hopf conjecture for $R^{3}$ remains unresolved.

Our counterexample is contained in the following theorem.

CountereXAMPle TheOREM. There is a conformal immersion of $R^{2}$ into $R^{3}$ with constant mean curvature $H \neq 0$ which is doubly-periodic with respect to a rectangle in $R^{2}$. If $w=u+i v=(u, v)$ represents a typical 
point in $R^{2}$ and we label points in $R^{3}$ by $\mathbf{x}=(x, y, z)$, then we construct $a$ function $\mathbf{x}(u, v)$ satisfying

$$
\begin{aligned}
& \text { (a) } \Delta \mathbf{x}=2 H\left(\mathbf{x}_{u} \wedge \mathbf{x}_{v}\right) \quad \text { some } H \neq 0 \\
& \text { (b) }\left|\mathbf{x}_{u}\right|=\left|\mathbf{x}_{v}\right|, \quad\left(\mathbf{x}_{u} \cdot \mathbf{x}_{v}\right)=0 \\
& \text { (c) }\left|\mathbf{x}_{u}\right| \neq 0 .
\end{aligned}
$$

These equations say that $\mathbf{x}(u, v)$ is an immersion of $R^{2}$ into $R^{3}$ with constant mean curvature, $H$. Finally, there are positive numbers $A, B$ with

$$
\mathbf{x}(u+A, v)=\mathbf{x}(u, v+B)=\mathbf{x}(u, v)
$$

for all $(u, v) \in R^{2}$. In fact, we produce a countable number of isometrically distinct immersions.

If $\mathbf{x}(u, v)$ is a solution to (1.1) (1.2), then we may write the first fundamental form $d s^{2}=E\left(d u^{2}+d v^{2}\right)$. Now set $E=\left(a^{2} / 4\right) e^{2 \omega}$ where $H=a^{-1} \neq 0$ then as we shall see in the next section, $\omega(u, v)$ will be a doubly-periodic solution of the P.D.E.

$$
\Delta \omega+\sinh \omega \cosh \omega=0
$$

where $\Delta \omega=\omega_{u u}+\omega_{v v}$ is the Laplace operator. Conversely, if $\omega$ is a doubly periodic solution to (1.3), then the Gauss and Mainardi-Codazzi Equations may be used to construct a mapping $\mathbf{x}(u, v)$ satisfying the constant mean curvature equations (1.1) and such that the lines of curvature are parallel to the coordinate axes in $R^{2}$. This mapping is generally not doubly periodic. These surfaces do possess some symmetry properties, however. We use a continuity argument to show that for certain rectangles $(A, B)$ the resulting surface is doubly periodic giving us our desired counterexample.

In §II we develop our construction procedure. In \$III we give the continuity argument leading to the existence of doubly periodic immersions. In §IV we discuss the needed results concerning solutions to the D. E. (1.3). In particular we will study "large" solutions to the D. E. of the form

$$
\Delta \omega+2 \lambda \sinh \omega=0, \quad \omega=0 \text { on } \partial \Omega
$$

where $\Omega$ is a rectangular domain. Our treatment is based on recent work of V. Weston [18] and J. L. Moseley [12, 13]. Weston studied large positive solutions to $\Delta \omega+\lambda e^{\omega}=0$ on a smooth domain $\Omega \subset R^{2}$ with $\omega=0$ on $\partial \Omega$. Subject to certain restrictions on $\Omega$ he showed the existence of a branch of "large" solutions parameterized by $\lambda$ as $\lambda \rightarrow 0$. Moseley [12] 
then adapted Weston's method to equations of the form

$$
\Delta \omega+\lambda\left[e^{\omega}+\sigma(w) e^{-\omega}\right]=0
$$

which includes the case of interest here. For us the domain is not smooth. In $\S I V$ we shall see that the Integral Equation method of Weston and Moseley will work for us.

Finally in $\S \mathrm{V}$, we shall discuss the shape of these surfaces and shall give some indication of their form.

II. The differential geometry. Much of the following development may be found in Eisenhart [4, p. 297] or in the lecture notes of H. Hopf [8]. Suppose that $\mathbf{x}: \Omega \subset R^{2} \rightarrow R^{3}$ is a conformal representation of a surface of constant mean curvature $H . \mathbf{x}(u, v)$ then satisfies (1.1) and the first fundamental form is given by

$$
\mathrm{I}=d \mathbf{x} \cdot d \mathbf{x}=E\left(d u^{2}+d v^{2}\right)
$$

where using the classical convention $E=G=\left|\mathbf{x}_{u}\right|^{2}$ and $F=\left(\mathbf{x}_{u} \cdot \mathbf{x}_{v}\right)=0$. The second fundamental form is given by

$$
\begin{gathered}
\mathrm{II}=-(d \mathbf{x} \cdot d \boldsymbol{\xi})=L d u^{2}+2 M d u d v+N d v^{2} \\
L=-\left(\mathbf{x}_{u} \cdot \boldsymbol{\xi}_{u}\right)=\left(\mathbf{x}_{u u} \cdot \xi\right) \\
M=-\left(\mathbf{x}_{u} \cdot \boldsymbol{\xi}_{v}\right)=-\left(\mathbf{x}_{v} \cdot \boldsymbol{\xi}_{u}\right)=\left(\mathbf{x}_{u v} \cdot \boldsymbol{\xi}\right) \\
N=-\left(\mathbf{x}_{v} \cdot \boldsymbol{\xi}_{v}\right)=\left(\mathbf{x}_{v v} \cdot \boldsymbol{\xi}\right) .
\end{gathered}
$$

Here $\xi=\left(\mathbf{x}_{u} \wedge \mathbf{x}_{v}\right) /\left|\mathbf{x}_{u} \wedge \mathbf{x}_{v}\right|$ is the unit normal vector with the orientation determined by the mapping $\mathbf{x}(u, v)$. For this mapping we have

$$
\begin{aligned}
& \text { (a) } K=k_{1} k_{2}=\left(L N-M^{2}\right) / E^{2} \\
& \text { (b) } H=\left(k_{1}+k_{2}\right) / 2=(L+N) / 2 E
\end{aligned}
$$

where $k_{1}, k_{2}$ are the principal curvatures, $K$ is the Gauss curvature, and $H$ is the mean curvature. For this system the Mainardi-Codazzi equations are

$$
\begin{aligned}
& \text { (a) } L_{v}-M_{u}=E_{v} H \\
& \text { (b) } M_{v}-N_{u}=-E_{u} H .
\end{aligned}
$$

However, $E H=(L+N) / 2$ so that the system (2.4) may be rewritten

$$
\begin{aligned}
& \text { (a) }[(L-N) / 2]_{u}+M_{v}=E H_{u} \\
& \text { (b) }[(L-N) / 2]_{v}-M_{u}=-E H_{v} \text {. }
\end{aligned}
$$

Therefore, if $H=$ constant we find that

$$
\phi(w)=[(L-N) / 2]-i M
$$


is a complex analytic function of $w=u+i v$, and it is easily checked that

$$
|\phi(w)|=\left|k_{1}-k_{2}\right| E / 2 .
$$

We assume that $\mathbf{x}(u, v)$ is an immersion so that $E>0$.

Now suppose further that $\mathbf{x}(u, v)$ is a doubly periodic immersion of $R^{2}$ into $R^{3}$. In this case $\phi(w)$ is a doubly periodic analytic function on $C$ and so is a constant. This leads to two possibilities.

Case $1 .|\phi(w)| \equiv 0$. Here we have $k_{1}=k_{2}$ so that the entire surface is umbilic. The only closed bounded surface with this property is the round sphere. We thus would have a conformal map of a torus $T^{2}$ onto a sphere $S^{2} \subset R^{3}$. This is impossible.

Case 2. $|\phi(w)|>0$. We now have $k_{1}-k_{2} \neq 0$ and the surface is free of umbilic points. The lines of curvature are given by the form

$$
-M d u^{2}+(L-N) d u d v+M d v^{2} \equiv 0 .
$$

But $L-N$ and $M$ are constant and not both zero. Therefore the lines of curvature are a family of mutually orthogonal straight lines in the $u-v$ plane.

If the lines of curvature happen to be parallel to the coordinate axes, then we have $M=0, k_{1}=L / E, k_{2}=N / E$ from which we obtain

$$
\xi_{u}=-k_{1} \mathbf{x}_{u}, \quad \xi_{v}=-k_{2} \mathbf{x}_{v} .
$$

We also note that in Case 2 the mean curvature $H \neq 0$ (there is no closed bounded minimal surface) and so by choice of orientation we may suppose $H$ is positive. We select $H=1 / 2$.

In conformal coordinates Gauss's Theorem Egregium may be written

$$
K=-\left[\left(E_{u} / E\right)_{u}+\left(E_{v} / E\right)_{v}\right] / 2 E .
$$

Suppose that the lines of curvature are parallel to the coordinate axes, that $k_{1}<k_{2}$, and that $k_{1}+k_{2}=2 H=1$. It follows that $-1<k_{1} / k_{2}<1$ and so there is a unique value $\omega$ with $k_{1}=C \sinh \omega, k_{2}=C \cosh \omega$ where $C=C(u, v)$ is to be determined. However $k_{1}+k_{2}=C[\cosh \omega+\sinh \omega]$ $=C e^{\omega}=1$ allows us to conclude that $C=e^{-\omega}$. Hence

$$
\begin{aligned}
& k_{1}=e^{-\omega} \sinh \omega<k_{2}=e^{-\omega} \cosh \omega \\
& K=e^{-2 \omega} \sinh \omega \cosh \omega .
\end{aligned}
$$

Since $\phi(w)$ is a constant it follows from (2.7) that $\left(k_{2}-k_{1}\right) E$ is constant and therefore $E=\lambda e^{2 \omega}$ for some constant $\lambda$. We substitute this into (2.10) and obtain

$$
\Delta \omega+\lambda \sinh \omega \cosh \omega=0
$$


By stretching the domain via a homothety we may force $\lambda$ to equal 1 , and so for suitably stretched coordinates we have

$$
\Delta \omega+\sinh \omega \cosh \omega=0 .
$$

We want to reverse this procedure. Start with a solution $\omega(u, v)$ of $(2.13)$ and from it construct an immersion of a surface with constant mean curvature $H=1 / 2$. This procedure is indicated in Eisenhart [4, p. 297].

The Reverse Procedure. Let $\Omega_{A B} \subset R^{2}$ be the open rectangular domain $(0, A) \times(0, B)$ and suppose $\omega(u, v)$ is a positive solution to the P.D.E. (2.13) which vanishes on the boundary. The following theorem applies.

THEOREM 2.1. Let $\omega(u, v) \in C^{2}\left(\Omega_{A B}\right) \cap C^{0}\left(\bar{\Omega}_{A B}\right)$ be a positive solution to the D.E.

$$
\omega_{u u}+\omega_{v v}+F(\omega)=0
$$

on $\Omega_{A B}$ which vanishes on the boundary, where $F(\omega)$ is an odd smooth function, $F(-\omega)=-F(\omega)$ and where we also assume that $F(\omega)$ is positive for $\omega$ positive. The following assertions are true.

(a) By odd reflection over adjacent rectangles $\omega(u, v)$ can be extended as a doubly periodic solution to (2.14) of class $C^{2}$ on $R^{2}$ satisfying

$$
\begin{aligned}
& \omega(u+2 A, v)=\omega(u, v+2 B)=\omega(u, v) \\
& \omega(-u, v)=\omega(u,-v)=-\omega(u, v)
\end{aligned}
$$

(b) The solution $\omega(u, v)$ is symmetric about the bisecting lines $u=A / 2$, $v=B / 2$.

$$
\begin{aligned}
& \omega(A-u, v)=\omega(u, B-v)=\omega(u, v) \\
& \omega(A / 2-u, v)=\omega(A / 2+u, v) \quad \text { etc. }
\end{aligned}
$$

(c) For a fixed $v, 0<v<B, \omega(u, v)$ is a strictly increasing function of $u, 0 \leq u \leq A / 2$, and for a fixed $u, 0<u<A, \omega(u, v)$ is a strictly increasing function of $v, 0 \leq v \leq B / 2$.

(d) $\omega_{v}(u, 0)$ is a strictly increasing function of $u, 0 \leq u \leq A / 2$ and $\omega_{u}(0, v)$ is a strictly increasing function of $v, 0 \leq v \leq B / 2$.

Proof. See Lemmas 4.5-4.6.

Suppose we have a smooth (analytic, in fact) doubly periodic solution to (2.13) satisfying the symmetry properties listed in Theorem 2.1. We 
recover the immersion $\mathbf{x}(u, v)$ as follows, where the constant mean curvature $H=1 / 2$. We set

$$
\begin{aligned}
E & =e^{2 \omega}, \quad d s^{2}=E\left(d u^{2}+d v^{2}\right) \\
k_{1} & =e^{-\omega} \sinh \omega, \quad k_{2}=e^{-\omega} \cosh \omega \\
L & =k_{1} E=e^{\omega} \sinh \omega \\
N & =k_{2} E=e^{\omega} \cosh \omega, \quad M=0 .
\end{aligned}
$$

We write

$$
\begin{aligned}
& K=k_{1} k_{2}=\left(L N-M^{2}\right) / E^{2} \text { and } \\
& H=(L+N) / 2 E=\left(k_{1}+k_{2}\right) / 2=1 / 2 .
\end{aligned}
$$

Our first and second fundamental forms are

$$
\begin{aligned}
d s^{2} & =e^{2 \omega}\left(d u^{2}+d v^{2}\right) \\
-d \mathbf{x} \cdot d \xi & =e^{\omega}\left[(\sinh \omega) d u^{2}+(\cosh \omega) d v^{2}\right] .
\end{aligned}
$$

It is straightforward to check that the Gauss Equation (2.10) and the Mainardi-Codazzi Equations (2.4-2.6) are satisfied. These equations (by a Theorem of Bonnet) see [3, p. 311] are precisely the integrability conditions that guarantee the existence of a mapping $\mathbf{x}(u, v)$ whose first and second fundamental forms are given by (2.18). If $E=G=e^{2 \omega}, F=0$ then the equations to be integrated may be written

$$
\begin{aligned}
\mathbf{x}_{u u} & =\omega_{u} \mathbf{x}_{u}-\omega_{v} \mathbf{x}_{v}+L \xi \\
\mathbf{x}_{u v} & =\omega_{v} \mathbf{x}_{u}+\omega_{u} \mathbf{x}_{v}+M \xi \\
\mathbf{x}_{v v} & =-\omega_{u} \mathbf{x}_{u}+\omega_{v} \mathbf{x}_{v}+N \xi \\
\xi_{u} & =-k_{1} \mathbf{x}_{u} \\
\xi_{v} & =-k_{2} \mathbf{x}_{v}
\end{aligned}
$$

where $k_{1}$ and $k_{2}$ are given by (2.17) and $\xi=\left(\mathbf{x}_{u} \wedge \mathbf{x}_{v}\right) /\left|\mathbf{x}_{u} \wedge \mathbf{x}_{v}\right|$. Our discussion gives us the followng theorem.

THEOREM 2.2. Let $\omega(u, v)$ be a solution to (2.13) on $R^{2}$.

Let the first and second fundamental forms be given by (2.18). There is a solution $\mathbf{x}(u, v)$ to the system (2.19) whose fundamental forms are given by (2.18). The lines of curvature are parallel to the coordinate axes and the principal curvatures are $k_{1}=e^{-\omega} \sinh \omega$ and $k_{2}=e^{-\omega} \cosh \omega$ with mean curvature $H=1 / 2$. The solution $\mathbf{x}(u, v)$ is unique to within a Euclidean motion in $R^{3}$. 
Proof. Follows from the previous discussion. This result may be found in Eisenhart [4].

Now suppose $\mathbf{x}(u, v)$ is the parametric surface of constant mean curvature $H=1 / 2$ obtained from Theorem 2.2. by integrating the system (2.19) where $\omega(u, v)$ is a solution to (2.13) on $R^{2}$ which is doubly periodic relative to the fundamental rectangle $[0,2 A] \times[0,2 B]$ satisfying the symmetry properties listed in Theorem 2.1. We are interested in the symmetry properties of $\mathbf{x}(u, v)$ itself.

THEOREM 2.3. Let $\mathbf{x}(u, v): R^{2} \rightarrow R^{3}$ be a parametric surface obtained by integrating the system (2.19), satisfying the conclusions of Theorem 2.2 where $\omega(u, v)$ is a solution to (2.13) and satisfying the symmetry conditions listed in Theorem 2.1. The surface $\mathbf{x}(u, v)$ possesses the following symmetry properties.

I. The curve $\mathbf{x}[(m+(1 / 2)) A$, $v]$ lies in a plane $\Pi_{m}(m$ is an integer $)$ whose normal vector is $\mathbf{x}_{u}[(m+(1 / 2)) A, v]$ and

$$
\mathbf{x}[(m+(1 / 2)) A-u, v]=\mathscr{R}_{m} \circ \mathbf{x}[(m+(1 / 2)) A+u, v]
$$

where $\mathscr{R}_{m}: R^{3} \rightarrow R^{3}$ is the reflection map about the plane $\Pi_{m}$. In particular this implies that

$$
\mathbf{x}(u, v)=\mathscr{R}_{0} \circ \mathbf{x}(A-u, v)=\mathscr{R}_{-1} \circ \mathbf{x}(-A-u, v) .
$$

The planes $\Pi_{m}$ are all parallel. Therefore $\mathbf{x}(u+2 A, v)=\mathbf{x}(u, v)+\mathbf{c}$ where $T(\mathbf{x})=\mathbf{x}+\mathbf{c}$ is a translation $T=\mathscr{R}_{0} \circ \mathscr{R}_{-1}$ in a direction normal to each $\Pi_{m}$ and carries $\Pi_{m}$ into $\Pi_{m+2}$. If $\mathbf{e}$ is a unit normal vector to $\Pi_{m}$, $\mathbf{e}=\mathbf{x}_{u}(A / 2,0) /\left|\mathbf{x}_{u}(A / 2,0)\right|$ then we may write

$$
\mathbf{x}(u+2 A, v)=\mathbf{x}(u, v)+\tau \mathbf{e}
$$

for some real number $\tau$.

II. $\mathbf{x}(u, l B)$ ( $l$ an integer) is a plane curve lying in a plane $\Gamma_{l}$ whose normal is given by $\xi(u, l B)=$ constant. In particular $\Gamma_{l} \perp \Pi_{m}$ for all $l, m$.

III. The curves $\mathbf{x}(m A, v)$ satisfy the identity $\mathbf{x}(m A, v)+\xi(m A, v)=\mathbf{c}_{m}$ where $\mathbf{c}_{m}$ is a constant vector. Thus $\mathbf{x}(m A, v) \subset S\left(\mathbf{c}_{m}, 1\right) \equiv$ the unit sphere with center $\mathbf{c}_{m}$. The centers $\mathbf{c}_{m}$ all lie on an axial line $l$ with direction parallel to the vector $\mathbf{e}$ given in $\mathrm{I}$, and $\mathbf{c}_{0} \mathbf{c}_{2}=\tau \mathbf{e}$.

IV. The curve $\mathbf{x}[u,(n+(1 / 2)) B]$ lies in a plane $\Omega_{n}$. This plane is orthogonal to each $\Pi_{m}$, its normal is given by $\mathbf{x}_{v}[u,(n+(1 / 2)) B]$, and each $\Omega_{n}$ contains the axial line l. As in I we have $\mathbf{x}(u, v)=\mathscr{W}_{0} \circ \mathbf{x}(u, B-v)=$ $\mathscr{W}_{-1} \circ \mathbf{x}(u, B-v)$ where $\mathscr{W}_{n}$ is the reflection in $R^{3}$ about the plane $\Omega_{n}$. It follows that

$$
\mathbf{x}(u, v+2 B)=\mathscr{R} \circ \mathbf{x}(u, v)
$$


where $\mathscr{R} \circ \mathbf{x}$ is a rotation through an angle $\theta$, with the line $l$ as the axis of rotation. A positive rotation is determined by the oriented normal $\mathbf{x}_{v}(u, B / 2)$ to $\Omega_{0}$, and $\boldsymbol{\theta}$ is the angle between the planes $\Omega_{0}$ and $\Omega_{2} . \mathscr{R} \circ \mathbf{x}=\mathscr{W}_{0} \circ \mathscr{W}_{-1} \circ \mathbf{x}$ in fact.

Proof. The theorem follows from the symmetry properties of the curvature function $\omega(u, v)$ as listed in Theorem 2.1. Since $M=0$ and $\omega_{u}(A / 2, v)=0$ we have from (2.19)

$$
\mathbf{x}_{u v}(A / 2, v)=\omega_{v}(A / 2, v) \mathbf{x}_{u}(A / 2, v) .
$$

From this we may conclude that $\mathbf{x}_{u}(A / 2, v)=\alpha(v) \mathbf{b}_{0}$ where $\alpha(v)$ is a positive scalar function and $\mathbf{b}_{0}$ is a constant vector, $\mathbf{b}_{0}=\mathbf{x}_{u}(A / 2,0)$ for example. It follows that $\mathbf{x}(A / 2, v)$ lies in a plane $\Pi_{0}$ with $\mathbf{x}_{u}(A / 2,0)=\mathbf{b}_{0}$ as a normal vector. Furthermore, from the symmetry properties (2.15) (2.16) of $\omega(u, v)$ it follows that by integrating the system (2.19) the surface $\mathbf{x}(u, v)$ will satisfy the symmetry property $\mathbf{x}(A / 2-u, v)=$ $\mathscr{R}_{0} \circ \mathbf{x}(A / 2+u, v)$ where $\mathscr{R}_{0}$ is the reflection about the plane $\Pi_{0}$.

Identical statements apply to the curves $\mathbf{x}[(m+(1 / 2)) A, v]$ with corresponding planes of symmetry $\Pi_{m}$ thus showing (2.20) and (2.21), and to the curves $\mathbf{x}[u,(n+(1 / 2)) B]$ contained in the planes of symmetry $\Omega_{n}$ orthogonal to $\Pi_{m}$.

Now look at the curve $\mathbf{x}(u, 0)$. We have $k_{1}(u, 0)=e^{-\omega} \sinh \omega=0$. Therefore, by (2.19), $\xi_{u}(u, 0)=\mathbf{0}$ or $\xi(u, 0)=$ constant. From this it follows that $\mathbf{x}(u, 0)$ is a curve lying in a plane $\Gamma_{0}$ which has $\xi(u, 0)$ as its normal vector. Thus $\Gamma_{0}$ is a tangent plane to the surface along the entire curve $\mathbf{x}(u, 0)$. Furthermore $\Gamma_{0}$ is orthogonal to the planes $\Pi_{m}$.

We now show that $\mathbf{x}_{u}(u, 0)$ is an even function of $u$. It will then follow that the planes $\Pi_{-1}$ and $\Pi_{0}$ are parallel, hence all $\Pi_{m}$ are parallel. Since $\xi(u, 0)$ is a constant unit vector we may suppose that $\xi(u, 0)=$ $(0,0,1)$, that $\mathbf{x}_{u}(u, 0)=(F(u), G(u), 0)$. Now $\left|\mathbf{x}_{u}(u, 0)\right|=\left|\mathbf{x}_{v}(u, 0)\right|=1$ so that $\mathbf{x}_{v}(u, 0)=(-G(u), F(u), 0)$ where $F^{2}+G^{2}=1$. From (2.19) we see that D.E. for $\mathbf{x}_{u u}$ is $\mathbf{x}_{u u}(u, 0)=-\omega_{v}(u, 0) \mathbf{x}_{v}(u, 0)$. This gives us the system of O.D.E.'s for $F$ and $G, F^{\prime}(t)=\omega_{v}(t, 0) G(t), G^{\prime}(t)=-\omega_{v}(t, 0) F(t)$ where $\omega_{v}(t, 0)$ is an odd function of $t$. Let $f(t)=F(-t), g(t)=G(-t)$. It is easy to check that the pair $(f(t), g(t))$ is a solution to the system if $(F(t), G(t))$ is a solution. Now $(f(0), g(0))=(F(0), G(0))$ and so by the uniqueness theorem for O.D.E.'s we conclude that $F(-t)=F(t)$ and $G(-t)=G(t)$ and so $\mathbf{x}_{u}(u, 0)$ is an even function of $u$.

Now consider the curve $\mathbf{x}(0, v)$. Here we have the D.E. $\xi_{v}(0, v)=$ $-k_{2}(0, v) \mathbf{x}_{v}(0, v)=-\mathbf{x}_{v}(0, v)$. From this we obtain $\mathbf{x}(0, v)+\xi(0, v)=\mathbf{c}_{0}$ $=$ constant. 
Therefore the curve $\mathbf{x}(0, v)$ lies on the unit sphere $S\left(\mathbf{c}_{0}, 1\right)$ with center $\mathbf{c}_{0}$ and radius 1 . The same result holds for the curve $\mathbf{x}(m A, v)$ which lies on the sphere of radius 1 and center $\mathbf{c}_{m}$.

Our discussion has shown that the planes $\Pi_{m}$ are parallel. We conclude that $\mathbf{x}(u+2 A, v)=\mathbf{x}(u, v)+\mathbf{c}$ where the translation vector $\mathbf{c}=\mathbf{c}_{2}-\mathbf{c}_{0}$.

It now follows that the planes $\Omega_{n}$ containing the curves $\mathbf{x}(u,(n+(1 / 2)) B]$ also contain the centers $\mathbf{c}_{m}$ of the spheres $S\left(\mathbf{c}_{m}, 1\right)$. As shown above we have

$$
\mathbf{x}(u, v)=\mathscr{W}_{0} \circ \mathbf{x}(u, B-v)=\mathscr{W}_{-1} \circ \mathbf{x}(u,-B-v)
$$

where $\mathscr{W}_{0}, \mathscr{W}_{-1}$ are reflections about planes $\Omega_{0}, \Omega_{-1}$ respectively. It follows that $\mathbf{x}(u, v+2 B)=\mathscr{R} \circ \mathbf{x}(u, v)$ where $\mathscr{R}=\mathscr{W}_{0} \circ \mathscr{W}_{-1}$ is a rotation through an angle $\theta$ about the axis $l$ where $l$ is the line determined by the centers of the spheres $S\left(\mathbf{c}_{m}, 1\right)$. We may choose the positive direction of rotation to be determined by the normal vectors $\mathbf{x}_{v}(u, B / 2)$ to $\Omega_{0}$.

Letting $l$ be the line containing the centers $\mathbf{c}_{m}$ with direction given by $\mathbf{x}_{u}(A / 2,0)$ we have shown that $\mathbf{x}(u+2 A, v)=\mathbf{x}(u, v)+\mathbf{c}$ where $\mathbf{c}=$ $\mathbf{c}_{2}-\mathbf{c}_{0}$ is parallel to $l$, and $\mathbf{x}(u, v+2 B)=\mathscr{R} \circ \mathbf{x}(u, v)$ where $\mathscr{R}$ is a rotation through an angle $\theta$ around the axial line $l$. This parametric surface will describe a closed surface if the translation constant $\mathbf{c}=\mathbf{0}$ and if the rotation angle is a rational multiple of $2 \pi$. We discuss this possibility in §III.

III. Existence of closed surfaces. Consider the set of solutions $\omega(u, v)$ to (2.13) positive on the interior of the rectangle $\Omega_{A B}=(0, A) \times$ $(0, B)$ and vanishing on the boundary. This is a two parameter family of functions, and by the discussion of §II each solution in the family allows us to generate an immersion $\mathbf{x}(u, v) R^{2} \rightarrow R^{3}$ of constant mean curvature.

At this point it is convenient to identify rectangular domains of similar shape by conformally mapping all similar rectangles onto a chosen rectangle from each class. This leaves us with a one parameter family of rectangular domains. The second parameter becomes an eigenvalue appearing in the transformed P.D.E.

LEMmA 3.1. Let $\phi: R^{2} \rightarrow R^{2}$ be the map $\left(u^{\prime}, v^{\prime}\right)=\phi(u, v)=$ $(u \sqrt{\lambda}, v \sqrt{\lambda})$. A function $W\left(u^{\prime}, v^{\prime}\right)$ is a solution to the P.D.E. $W_{u^{\prime} u^{\prime}}+W_{v^{\prime} v^{\prime}}$ $+h(W)=0$ for some $C^{2}-$ function $h(W)$, iff $W(u, v) \equiv W \circ \Phi(u, v)$ is a solution to $\Delta W+\lambda h(W)=W_{u u}+W_{v v}+\lambda h(W) \equiv 0$. 
Proof. Immediate.

We shall select rectangles of various shapes by the Schwartz-Christoffel transformation of a rectangle onto the unit disk.

The Schwartz-Christoffel Map. For $0<\alpha<\pi / 2$ the transformation

$$
\begin{aligned}
w & =f(z, \alpha)=f(z)=\int_{0}^{z} \frac{d t}{\sqrt{\left(t^{2}-e^{2 i \alpha}\right)\left(t^{2}-e^{-2 i \alpha}\right)}} \\
& =\int_{0}^{z} \frac{d t}{\sqrt{t^{4}-2(\cos 2 \alpha) t^{2}+1}}
\end{aligned}
$$

is a conformal map of the unit disk $D$ in the $z$-plane onto the rectangle $\Omega(\alpha)$, centered about the origin and symmetric with respect to the coordinate axes. The points $z= \pm e^{i \alpha}, \pm e^{-i \alpha}$ are mapped onto the vertices of the rectangle. We note that $f\left(e^{i \alpha}\right)=(A(\alpha), B(\alpha))$

$$
\begin{aligned}
& \text { (a) } A(\alpha)=\int_{0}^{1} \frac{d t}{\sqrt{t^{4}-2(\cos 2 \alpha) t^{2}+1}} \\
& \text { (b) } B(\alpha)=\int_{0}^{1} \frac{d t}{\sqrt{t^{4}+2(\cos 2 \alpha) t^{2}+1}}
\end{aligned}
$$

with limit ${ }_{\alpha \rightarrow 0} A(\alpha)=+\infty$ and limit ${ }_{\alpha \rightarrow 0} B(\alpha)=\pi / 2$. (See Figure 1.) We shall write $w=f(z, \alpha)$ if the dependence of the mapping on $\alpha$ is being considered. We note that the inverse map $z=g(w, \alpha)$ to $w=f(z, \alpha)$ has an extension analytic in $w$ to all of $C$ while the map $w=f(z, \alpha)$ is analytic in $z$ on the open unit disk $D$, continuous on $\bar{D}$, and of class $\operatorname{Lip}\left(\frac{1}{2}, \bar{D}\right)$ (i.e. Lipschitz (or) Hölder continuous with Lipschitz exponent 1/2) where the Lipschitz constant depends continuously on the parameter $\alpha$.

In §IV we shall prove the following theorem.

THEOREM 3.1. There is a real valued function $\Psi(z, \alpha, \lambda)$ such that for any $\alpha_{1}, \alpha_{2}$ with $0<\alpha_{1}<\alpha_{2}<\pi / 2$ there exists $\tilde{\lambda}=\tilde{\lambda}\left(\alpha_{1}, \alpha_{2}\right)>0$ the domain of $\Psi$ will include the set $\bar{D} \times\left[\alpha_{1}, \alpha_{2}\right] \times[0, \tilde{\lambda}]$ with the following properties.

1. $\Psi(z, \alpha, \lambda)$ is continuous in its variables except when $z=0, \lambda=0$. For $\lambda=0$ we have $\Psi(z, \alpha, 0)=4 \ln (1 /|z|)$.

2. If we set $\Sigma(w, \alpha, \lambda)=\Psi[g(w, \alpha), \alpha, \lambda]$ where $z=g(w, \alpha)$ is the inverse of the Schwartz-Christoffel map, then $\Sigma(w, \alpha, \lambda)$ is a positive solution to

$$
\Delta \Sigma+\lambda\left(e^{\Sigma}-e^{-\Sigma}\right)=\Delta \Sigma+2 \lambda \sinh \Sigma=0
$$


on the rectangle $\Omega(\alpha)$, and vanishing on the boundary. This solution necessarily has all of the symmetry properties described in Lemma 2.1 and so has $a($ real $)$ analytic extension to the entire $w$-plane. For $\lambda=0, \Sigma(w, \alpha, 0)=$ $4 \ln (1 /|g(w, \alpha)|)$ is harmonic except at $w=0$ and at the reflection points with respect to $\Omega(\alpha)$ when extended analytically to $R^{2}$.

3. The partial derivatives $\Sigma_{u}, \Sigma_{v}$ are continuous funtions of $(w, \alpha, \lambda)$ on any domain $\overline{\Omega(\alpha)} \times\left[\alpha_{1}, \alpha_{2}\right] \times[0, \tilde{\lambda}]$ except for $(w, \alpha, \lambda)=(0, \alpha, 0)$.

\section{Proof. To be given in §IV.}

Each solution $\Sigma(w, \alpha, \lambda)$ to (3.3) defined on $\Omega(\alpha)$ depending continuously on $\alpha, \lambda$ as stated in Theorem 3.1 allows us to construct an immersion $\mathrm{x}(u, v): R^{2} \rightarrow R^{3}$ of a surface with constant mean curvature $H=1 / 2$ using the recipe of $\S$ II. Let $\Sigma\left(u^{\prime}, v^{\prime}\right)$ be a solution to (3.3) on the rectangle $\Omega(\alpha)$, set $\left(u^{\prime}, v^{\prime}\right)=\phi(u, v)=(u / \sqrt{2 \lambda}, v / \sqrt{2 \lambda})$ and define $\sigma(u, v)=$ $\Sigma \circ \phi(u, v)$. It follows from Lemma 3.1 that $\sigma(u, v)$ is a solution to

$$
\Delta \sigma+\sinh \sigma=0
$$

which is positive on the rectangle $\Omega(\alpha, \lambda)$ and vanishing on the boundary. $\Omega(\alpha, \lambda)$ is the rectangle symmetric with respect to the coordinate axes with first quadrant vertex at $(\sqrt{2 \lambda} A(\alpha), \sqrt{2 \lambda} B(\alpha))$. Finally we set $\omega(u, v)$ $=\sigma(u, v) / 2$ and find that $\omega(u, v)$ is a positive solution to

$$
\Delta \omega+\sinh \omega \cosh \omega=0 \text { on } \Omega(\alpha, \lambda) .
$$

We are now in a position to construct the immersion $\mathbf{x}(u, v)$ [which depends on $\lambda$ and $\alpha$ as well] using the method of $\S I I$. The fundamental rectangle $\Omega(\alpha, \lambda)$ is presently centered about the origin. A simple translation will put it into the standard position of $\S I I$ where two of the sides lay on the coordinate axes.

Label the vertices of $\Omega(\alpha, \lambda)$ by $(A(\alpha, \lambda), B(\alpha, \lambda))$ where $A(\alpha, \lambda)$ $=\sqrt{2 \lambda} A(\alpha), B(\alpha, \lambda)=\sqrt{2 \lambda} B(\alpha)$ and suppose we have constructed our surface $\mathbf{x}(u, v)$ with the fundamental rectangle as $\Omega(\alpha, \lambda)$ centered about the origin. For this surface we have the parallel planes $\Pi_{0}$ containing the curve $\mathbf{x}(0, v)$ with normal $\mathbf{x}_{u}(0, v)$ and $\Pi_{1}$ containing the curve $\mathbf{x}(2 A(\alpha, \lambda), v)$ parallel to $\Pi_{0}$ (See Theorem 2.3, keeping in mind that we have shifted the fundamental domain). [See Figure 2.]

We wish to identify values $(\alpha, \lambda)$ for which $\Pi_{0}=\Pi_{1}$. To do this it is convenient to look at the expanded surface $\mathbf{y}(u, v)=\mathbf{x}(u, v) / \sqrt{2 \lambda}$ and to consider these surfaces as functions of $\left(u^{\prime}, v^{\prime}\right)$ relative to the fundamental rectangle $\Omega(\alpha)$.

$$
\mathbf{y}\left(u^{\prime}, v^{\prime}\right)=\mathbf{x} \circ \phi^{-1}\left(u^{\prime}, v^{\prime}\right) / \sqrt{2 \lambda}
$$


where $\left(u^{\prime}, v^{\prime}\right)=\phi(u, v)=(u / \sqrt{2 \lambda}, v / \sqrt{2 \lambda})$. The surface $\mathbf{y}\left(u^{\prime}, v^{\prime}\right)$ has mean curvature $H=\sqrt{2 \lambda} / 2$ and so is being flattened out as $\lambda \rightarrow 0$. The fundamental equations (2.19) for the surface $\mathbf{y}\left(u^{\prime}, v^{\prime}\right)$ become the following (where we now drop the cumbersome $\left(u^{\prime}, v^{\prime}\right)$ notation and replace it by $(u, v))$.

$$
\begin{aligned}
\mathbf{y}_{u u} & =W_{u} \mathbf{y}_{u}-W_{v} \mathbf{y}_{v}+\tilde{L} \xi \\
\mathbf{y}_{u v} & =W_{v} \mathbf{y}_{u}+W_{u} \mathbf{y}_{v}+\tilde{M} \xi \\
\mathbf{y}_{v v} & =-W_{u} \mathbf{y}_{u}+W_{v} \mathbf{y}_{v}+\tilde{N} \xi \\
\boldsymbol{\xi}_{u} & =-\tilde{k}_{1} \mathbf{y}_{u} \\
\boldsymbol{\xi}_{v} & =-\tilde{k}_{2} \mathbf{y}_{v} .
\end{aligned}
$$

Here $E=G=e^{2 W}, F=0$

$$
\begin{aligned}
\tilde{k}_{1}=\sqrt{2 \lambda} k_{1}=\sqrt{2 \lambda} e^{-W} \sinh W, & \tilde{k}_{2}=\sqrt{2 \lambda} e^{-W} \cosh W \\
\tilde{L}=\sqrt{2 \lambda} L=\sqrt{2 \lambda}\left(e^{W} \sinh W\right), & \tilde{M}=0, \tilde{N}=\sqrt{2 \lambda} N .
\end{aligned}
$$

Here $W \equiv \Sigma / 2$ and so by (3.3) $W$ is a solution to

$$
\Delta W+2 \lambda \sinh W \cosh W=0
$$

and $\operatorname{limit}_{\lambda \rightarrow 0} W(u, v, \alpha, \lambda)=2 \ln (1 /|g(w, \alpha)|)$, using Theorem 3.1. Here $W(u, v, \alpha, \lambda)$ is a positive solution of (3.8) on the fundamental rectangle $\Omega(\alpha)$ vanishing on the boundary. It has been extended smoothly to all of $R^{2}$, satisfying the symmetry properties listed in Theorem 3.1. Also $W(u, v, \alpha, \lambda), W_{u}(u, v, \alpha, \lambda), W_{v}(u, v, \alpha, \lambda)$ are continuous in its variables down to the limit $\lambda=0$.

Now select that solution $\mathbf{y}(u, v, \alpha, \lambda)$ to (3.7) for which at $(u, v)=$ $(0,-B(\alpha)), \mathbf{y}=\mathbf{0}, \mathbf{y}_{u}=(1,0,0), \mathbf{y}_{v}=(0,-1,0), \xi=(0,0,-1)$. Because of the continuous dependence of $W, W_{u}, W_{v}$ on $(\alpha, \lambda)$ the solutions $\mathbf{y}(u, v, \alpha, \lambda)$ will have a continuous dependence on $(\alpha, \lambda)$ as solutions to (3.7) down to the limit $\lambda=0$.

Our choice of initial conditions for $\mathbf{y}(u, v, \alpha, \lambda)$ causes the plane $\Pi_{0}^{\prime}$ containing the curve $\mathbf{y}(0, v, \alpha, \lambda)$ to be the plane $x=0$, while $\Pi_{1}^{\prime}$ is that plane parallel to $\Pi_{0}^{\prime}$ containing the curve $\mathbf{y}(2 A(\alpha), \mathrm{v}, \alpha, \lambda)$.

Definition 3.1. We define a function $S(\alpha, \lambda)$ by setting $\Pi_{1}^{\prime}$ to be the plane $x=2 S=2 S(\alpha, \lambda)$ which is parallel to $\Pi_{0}^{\prime}$. We then have $2 S=$ $\tau / \sqrt{2 \lambda}$ where $\tau$ is the directed distance between the planes $\Pi_{0}$ and $\Pi_{1}$ for the original surface.

THEOREM 3.2. The function $S(\alpha, \lambda)$ which is defined on an open set $U$ in the $(\alpha, \lambda)$ plane (where for each $0<\alpha_{1}<\alpha_{2}<\pi / 2$ there exists $\tilde{\lambda}\left(\alpha_{1}, \alpha_{2}\right)$ $>0$ such that $\left.\left[\alpha_{1}, \alpha_{2}\right] \times(0, \tilde{\lambda}] \subset U\right)$ has a continuous extension to $0<\alpha<$ $\pi / 2, \lambda=0 . S(\alpha, 0)$ is a strictly increasing function on the interval $(0, \pi / 2)$ 
with limit $S(\alpha, 0)=-\infty$ as $\alpha \rightarrow 0^{+}$and $S(\alpha, 0)$ positive for $\alpha>\pi / 4$. In particular there is exactly one value $\alpha^{*}, 0<\alpha^{*}<\pi / 4$ with $S\left(\alpha^{*}, 0\right)=0$.

Proof. From Theorem 3.1 we know that the functions $W(u, v, \alpha, \lambda)$, $W_{u}(u, v, \alpha, \lambda), W_{v}(u, v, \alpha, \lambda)$ are continuous in a horizontal strip $-3 B(\tilde{\alpha}) / 2<v<-B(\tilde{\alpha}) / 2$ and for $|\alpha-\tilde{\alpha}|<\sigma, 0<\lambda<\lambda_{0}$ for some $\lambda_{0}>0$ and $\sigma>0$, with $W(u, v, \alpha, 0)=2 \ln (1 /|g(w, \alpha)|)$. It follows that the functions $\mathbf{y}(u, v, \alpha, \lambda)$ solving the system (3.7) with initial conditions $\mathbf{y}=\mathbf{0}, \mathbf{y}_{u}=(1,0,0), \mathbf{y}_{v}=(0,-1,0), \xi=(0,0,-1)$ at $(u, v)=(0,-B(\alpha))$ are continuous in all of its variables along with the first and second partial derivatives with respect to $u, v$.

We consider the curve $y(u,-B(\alpha), \alpha, \lambda)$. By Theorem 2.3(III) this is a plane curve lying in the plane $\Gamma_{0}$ which by our choice of initial conditions is the plane $z=0 . \Gamma_{0}$ is a tangent plane to the surface with $\xi=(0,0,-1)$ the oriented unit normal.

Write $\mathbf{y}(u,-B(\alpha), \alpha, \lambda)=\langle F(u, \alpha, \lambda), G(u, \alpha, \lambda), 0\rangle$. From our initial conditions we have $F(0, \alpha, \lambda)=G(0, \alpha, \lambda)=0, F_{u}(0, \alpha, \lambda)=1$, $G_{u}(0, \alpha, \lambda)=0$, and $F(2 A(\alpha), \alpha, \lambda)=2 S(\alpha, \lambda)$. However, by the symmetry of the surface we also have $S(\alpha, \lambda)=F(A(\alpha), \alpha, \lambda)$. Clearly, $S(\alpha, \lambda)$ is a continuous function on the set $U \cup\{(\alpha, \lambda) \mid 0<\alpha<\pi / 2, \lambda=0\}$. It remains to investigate the function $S(\alpha, 0)$.

We look at the map $\mathbf{y}(u, v) \equiv \mathbf{y}(u, v, \alpha, 0)$ when $\lambda=0$ and write $\mathbf{y}(u,-B(\alpha))=\langle x(u), y(u)\rangle=\langle F(u), G(u)\rangle$. Let $\phi(u)$ be the angle of inclination of this curve. I claim that $\phi=2 \theta$ where $\theta$ is the angle on the unit circle in the $z$-plane measured from the point $x=-i$ to the image $g(u-B(\alpha) i)=e^{i[\theta-\pi / 2]}$ the inverse of the Schwartz-Christoffel mapping. (See Figure 3.)

To see this we write $w=u+i v$ and let $h(w)=x(w)+i y(w)$ where $\mathbf{y}(w, \alpha, 0)=(x(w, \alpha), y(w, \alpha), 0)$ and we have suppressed the dependance of $h$ on $\alpha$. The system (3.7) when $\lambda=0$ may be written in the complex form as

$$
h^{\prime \prime}(w)=\delta(w) h^{\prime}(w)
$$

where $\delta(w)=W_{u}-i W_{v}$ and $W(w, \alpha)=2 \ln (1 /|g(w, \alpha)|)$ so $\delta(w)$ is a complex analytic function of $w$. It follows that $\delta(w)=-2 g^{\prime}(w) / g(w)=$ $d(-2 \ln (g(w))) / d w$. An integration of (3.9) gives $h^{\prime}(w)=K /[g(w)]^{2}$. But $h^{\prime}(-B(\alpha) i)=1$ and $g(-B(\alpha) i)=-i$ whence $K=-1$

$$
h^{\prime}(w)=-1 / g^{2}(w) \text {. }
$$

By definition $g(u-B(\alpha) i)=e^{i(\theta-\pi / 2)}$ from which we obtain

$$
h^{\prime}(u-B(\alpha) i)=-1 / e^{2 i \theta-\pi \imath}=e^{-2 \imath \theta} .
$$


But $h^{\prime}(u-B i)=x^{\prime}(u)-i y^{\prime}(u)=e^{-i \phi}$ since $\left|\mathbf{y}_{u}(u,-B(\alpha))\right|^{2}=E=1$. Since $\phi(0)=\theta(0)=0$ we conclude that $\phi=2 \theta$.

Along the line $v=-B(\alpha)$ the function $W(u, v, \alpha, \lambda)$ solving (3.8) vanishes. This implies that along $v=-B(\alpha),|d \mathbf{y} / d u|=\sqrt{E}=1$. We have $\mathbf{y}(u,-B(\alpha), \alpha, 0)=\langle x(u), y(u), 0\rangle$. If we parameterize this curve by arc length we find

$$
\begin{aligned}
& x(\tilde{s})=\int_{0}^{\tilde{s}}\left(\frac{d x}{d s}\right) d s=\int_{0}^{\tilde{s}} \cos \phi(s) d s=\int_{0}^{\tilde{s}} \cos (2 \theta(s)) d s \\
& y(\tilde{s})=\int_{0}^{\tilde{s}} \sin \phi(s) d s=\int_{0}^{\tilde{s}} \sin (2 \theta(s)) d s .
\end{aligned}
$$

We now introduce the angle $\theta$ as parameter (3.11)

$$
x(\tilde{\theta})=\int_{0}^{\tilde{\theta}} \cos (2 \theta) \cdot\left(\frac{d s}{d \theta}\right) d \theta .
$$

From the Schwartz-Christoffel map (3.1) we have

$$
u+i v=w=\int_{0}^{z} \frac{d t}{\sqrt{t^{4}-2(\cos 2 \alpha) t^{2}+1}},
$$

where we shall now set $z=-i e^{\imath \theta}$. If we also put $v=-B(\alpha)$ then we obtain $d u / d \theta=e^{\imath \theta} /\left[e^{4 i \theta}+2(\cos 2 \alpha) e^{2 \imath \theta}+1\right]^{1 / 2}=(2 \cos 2 \theta+2 \cos 2 \alpha)^{-1 / 2}$.

Substitute this into (3.11) recalling that $d s / d u=1$ and we find

$$
\begin{aligned}
& x(\tilde{\theta})=\int_{0}^{\tilde{\theta}} \frac{\cos 2 \theta}{(2 \cos 2 \theta+2 \cos 2 \alpha)^{1 / 2}} d \theta \\
& y(\tilde{\theta})=\int_{0}^{\tilde{\theta}} \frac{\sin 2 \theta}{(2 \cos 2 \theta+2 \cos 2 \alpha)^{1 / 2}} d \theta .
\end{aligned}
$$

Now $S(\alpha, 0)=x(\pi / 2-\alpha)=\hat{S}(\beta)$ where we set $\beta=\pi / 2-\alpha$.

$$
S(\alpha, 0)=\int_{0}^{\beta} \frac{\cos 2 \theta}{(2 \cos 2 \theta-2 \cos 2 \beta)^{1 / 2}} d \theta=\hat{S}(\beta) .
$$

We need to show that $\hat{S}(\beta)$ is strictly decreasing in $\beta$, that limit $\hat{S}(\beta)=$ $-\infty$ as $\beta \rightarrow \pi / 2$, and $\hat{S}(\beta)$ is positive for $\beta<\pi / 4$.

If we introduce a new variable of integration by setting $\sin \theta=$ $\sin \beta \sin t$, where $0<\theta<\beta$ and write $k=\sin \beta$ we obtain

$$
\begin{aligned}
\hat{S}(\beta)= & \int_{0}^{\pi / 2}\left(1-k^{2} \sin ^{2} t\right)^{1 / 2} d t \\
& -\frac{1}{2} \int_{0}^{\pi / 2}\left(1-k^{2} \sin ^{2} t\right)^{-1 / 2} d t .
\end{aligned}
$$


The first integral is a decreasing function of $\beta$ while the second is increasing in $\beta$. Therefore, $\hat{S}(\beta)$ is a strictly decreasing function of $\beta$, $0 \leq \beta<\pi / 2$.

From (3.13) we see that for $\beta \leq \pi / 4, S(\alpha, 0)$ is positive. For $\beta=$ $\pi / 2(\alpha=0)$ the limiting trace curve $\langle x(\theta), y(\theta)\rangle$ is given by

$$
\text { (a) } \begin{aligned}
x(\tilde{\theta}) & =\int_{0}^{\tilde{\theta}} \frac{\cos 2 \theta}{2 \cos \theta} d \theta \\
& =\frac{1}{2}[2 \sin \tilde{\theta}-\ln |\sec \tilde{\theta}+\tan \tilde{\theta}|] \\
\text { (b) } y(\theta) & =\int_{0}^{\tilde{\theta}} \frac{\sin 2 \theta}{2 \cos \theta} d \theta=1-\cos \tilde{\theta} .
\end{aligned}
$$

Thus we see $\hat{S}(\pi / 2)=\operatorname{limit} x(\tilde{\theta})=-\infty$ as $\tilde{\theta} \rightarrow \pi / 2$.

Let $\alpha^{*}, 0<\alpha^{*}<\pi / 4$, be the unique $\alpha$ with $S\left(\alpha^{*}, 0\right)=0$. By continuity of the function $S(\alpha, \lambda)$ there is a closed rectangle $\left[\alpha_{1}, \alpha_{2}\right] \times[0, \tilde{\lambda}]$ with $\alpha_{1}<\alpha^{*}<\alpha_{2}$ and $\tilde{\lambda}>0$ so that $S\left(\alpha_{1}, \lambda\right)<0$ and $S\left(\alpha_{2}, \lambda\right)>0$ for $0 \leq \lambda$ $\leq \tilde{\lambda}, S\left(\alpha^{*}, 0\right)=0$, and $S(\alpha, 0)$ increasing for $\alpha_{1} \leq \alpha \leq \alpha_{2}$.

LEMma 3.2. Let $X$ be the set of $(\alpha, \lambda)$ in the closed rectangle $\left[\alpha_{1}, \alpha_{2}\right] \times$ $[0, \tilde{\lambda}]$ for which $S(\alpha, \lambda)=0$, where $S$ is a continuous function, $S\left(\alpha_{1}, \lambda\right)<0$, $S\left(\alpha_{2}, \lambda\right)>0$, and for exactly one $\alpha^{*}, \alpha_{1}<\alpha^{*}<\alpha_{2}$ we have $S\left(\alpha^{*}, 0\right)=0$. Let $Y$ be that component of $X$ which contains $\left(\alpha^{*}, 0\right)$. $Y$ is a connected set which separates the set $\left\{\alpha_{1}\right\} \times[0, \tilde{\lambda}]$ where $S<0$ from the set $\left\{\alpha_{2}\right\} \times[0, \tilde{\lambda}]$ where $S>0$. In particular, for any $\lambda^{\prime} \in[0, \tilde{\lambda}], Y \cap\left\{(\alpha, \lambda) \mid \lambda=\lambda^{\prime}\right\}$ is not empty.

Proof. Extend the domain of $S$ to the rectangle $\left[\alpha_{1}, \alpha_{2}\right] \times[-\tilde{\lambda}, \tilde{\lambda}]$ by setting $S(\alpha,-\lambda)=S(\alpha, \lambda)$. By identifying the points $(\alpha, \tilde{\lambda})$ with $(\alpha,-\tilde{\lambda})$ we may assume that $S$ is continuous on a cylinder. By extending the domain of $S$ slightly to the left of $\alpha_{1}$ and slightly to the right of $\alpha_{2}$ we may assume that $S$ is constant in $\lambda$ on each end of the cylinder. Identify the left and right hand ends of the cylinder with the south and north poles of the sphere $S^{2}$ and we see that our Lemma is equivalent to the following assertion.

Assertion. Let $f$ be a continuous real valued function of the sphere $S^{2}$. Suppose $f<0$ at the south pole and $f>0$ at the north pole. 
Let $A$ be the open component of $f<0$ containing the south pole and $B$ be the open component of $f>0$ containing the north pole. There is a connected subset $X$ of $f^{-1}(0)$ which separates $A$ from $B$.

Sketch of proof. Let $O$ be that component of $(\bar{A})^{c}$ which contains the north pole, $\infty$. Clearly $\infty \in B \subset O$ and $O$ is an open connected set since any component of an open set is open.

The following assertions can now be established in a fairly straightforward manner.

(i) $\partial O=\bar{O} \cap \bar{A}$, hence $f=0$ on $\partial O$.

(ii) $O$ is simply connected

(iii) $\partial O$ is connected. It now follows that $X \equiv \partial O$ is a connected subset of $f^{-1}(0)$ which separates $A$ from $B$.

Note. In $\S I V$ it will be shown that the function $S(\alpha, \lambda)$ is continuously differentiable for $\lambda$ positive. If this property were to persist down to the boundary $\lambda=0$, then it would follow that $X=S^{-1}\{0\}$ is the graph of a differentiable function $\alpha=\alpha(\lambda)$ with $\alpha(0)=\alpha^{*}$ since $S_{\alpha}$ would be positive in a neighborhood of $\left(\alpha^{*}, 0\right)$.

Now go back to the surface $\mathbf{x}(u, v, \alpha, \lambda)$ as constructed in Theorem 2.3 with the fundamental rectangle $\Omega(\alpha, \lambda)$ lying in the first quadrant and vertex at $(A, B)=(2 \sqrt{2 \lambda} A(\alpha), 2 \sqrt{2 \lambda} B(\alpha))$. For $(\alpha, \lambda)$ with $S(\alpha, \lambda)=0$ the surface closes up in the $u$-direction, the planes $\Pi_{m}$ are all identical, and $\mathbf{x}(u+2 A, v)=\mathbf{x}(u, v)$. From Theorem 2.3-IV we know that there is an axial line $l$ and an angle of rotation $2 \theta$ so that $\mathbf{x}(u, v+2 B)=$ $\mathscr{R} \circ \mathbf{x}(u, v)$ where $\mathscr{R}$ is a rotation through an angle $2 \theta$ about the axis $l$. This angle $2 \theta$ is a continuous function of $(\alpha, \lambda)$ for $\lambda>0$. We can measure this angle by examining the curve $\mathbf{x}(0, v)$.

Instead of $\mathbf{x}(u, v, \alpha, \lambda)$ it is more convenient to look at the expanded surface $\mathbf{y}(u, v, \alpha, \lambda)=\mathbf{x}(u, v, \alpha, \lambda) / \sqrt{2 \lambda}$ with the domain also blown up by the same factor. This surface $\mathbf{y}(u, v, \alpha, \lambda)$ will satisfy the system (3.7). Let us assume, as before, that the fundamental domain $\Omega(\alpha)$ is centered at the origin, with sides parallel to the coordinate axes and first quadrant vertex at $(A(\alpha), B(\alpha))$. Let initial conditions for $\mathbf{y}(u, v, \alpha, \lambda)$ be chosen so that for $u=A(\alpha), v=0$ we have $\mathbf{y}=\mathbf{0}, \mathbf{y}_{u}=(1,0,0), \mathbf{y}_{v}=(0,-1,0)$, $\xi=(0,0,-1)$.

The image of the line $v=0$ under the mapping $\mathbf{y}$ lies in a plane $\Omega_{0}^{\prime}$ while the image of the line $v=2 B(\alpha)$ lies in a plane $\Omega_{1}^{\prime}$. The two planes intersect on the axial line $l^{\prime}$. The curve $\mathbf{y}(A(\alpha), v, \alpha, \lambda)$ lies on a sphere with center $\mathbf{c}_{0}^{\prime} \in l^{\prime}$ and radius $(1 / \sqrt{2 \lambda}) . \theta(\alpha, \lambda)$ is the angle between $\Omega_{0}^{\prime}$ and $\Omega_{1}^{\prime}$. 
Theorem 3.3. For any $\alpha_{1}, \alpha_{2}$ with $0<\alpha_{1}<\alpha_{2}<\pi / 2$ and $0<\lambda<$ $\tilde{\lambda}\left(\alpha_{1}, \alpha_{2}\right)$ the function $G(\alpha, \lambda) \equiv \theta(\alpha, \lambda) / \sqrt{2 \lambda}$ extends continuously to $\left[\alpha_{1}, \alpha_{2}\right] \times[0, \tilde{\lambda})$ with $G(\alpha, 0)$ given by

$$
G(\alpha, 0)=2 \int_{0}^{\alpha} \frac{\cos 2 \theta d \theta}{\sqrt{2 \cos 2 \theta-2 \cos 2 \alpha}} .
$$

Proof. The proof is analogous to that of Theorem 3.2. The curve $\mathbf{y}(A(\alpha), v, \alpha, \lambda), 0 \leq v \leq B(\alpha)$ lies on the sphere $S\left[\mathbf{c}_{0}^{\prime}, 1 / \sqrt{2 \lambda}\right]$. The point $\mathbf{y}(A(\alpha), B(\alpha), \alpha, \lambda)$ determines a plane $\Omega^{\prime}$ containing the line $l^{\prime}$ which meets the plane $\Omega_{0}^{\prime}$ at the angle $\theta(\alpha, \lambda) / 2$.

The limit $\lambda \rightarrow 0$ of the curve $\mathbf{y}(A(\alpha), v, \alpha, \lambda)$ is the plane curve $\mathbf{y}(A(\alpha), v, \alpha, 0) \equiv\langle f(A(\alpha), v, \alpha), g(A(\alpha), v, \alpha), 0\rangle$ where $\langle f(u, v, \alpha)$, $g(u, v, \alpha), 0\rangle$ is the limiting surface, using (3.9) as discussed in the proof of Theorem 3.2. We obtain

$$
\operatorname{limit}_{\lambda \rightarrow 0} \theta(\alpha, \lambda) \cdot[1 / \sqrt{2 \lambda}]=2 g(A(\alpha), B(\alpha), \alpha) .
$$

By essentially repeating the calculations of (3.10)-(3.14) in the proof of Theorem 3.2, we find

$$
g(A(\alpha), B(\alpha), \alpha)=\int_{0}^{\alpha} \frac{\cos 2 \theta d \theta}{\sqrt{2 \cos 2 \theta-2 \cos 2 \alpha}},
$$

giving us (3.16).

Since $\mathbf{y}(u, v, \alpha, \lambda)$ depends continuously on its variables down to $\lambda=0$, we see that $G(\alpha, \lambda) \equiv \theta(\alpha, \lambda) / \sqrt{2 \lambda}$ is continuous on any rectangle $\left[\alpha_{1}, \alpha_{2}\right] \times[0, \lambda]$, with $G(\alpha, 0)$ given by (3.16).

The parametric surface will define a closed surface if the function $S(\alpha, \lambda)$ equals zero and the rotation function $\theta(\alpha, \lambda)$ is a rational part of $\pi$.

Let $\left[\alpha_{1}, \alpha_{2}\right] \times[0, \tilde{\lambda}]$ with $0<\alpha_{1}<\alpha_{2}<\pi / 2$ and $\tilde{\lambda}\left(\alpha_{1}, \alpha_{2}\right)>0$ be a rectangle as described in Theorem 3.2 and Lemma 3.2 with $\alpha_{1}<\alpha^{*}<\alpha_{2}$ and $S\left(\alpha^{*}, 0\right)=0$. Since $\alpha^{*}<\pi / 4$ we may suppose that our rectangle about $\left(\alpha^{*}, 0\right)$ is chosen so that $G(\alpha, \lambda)$ is positive on this rectangle. It follows that the angle function $\theta(\alpha, \lambda)=\sqrt{2 \lambda} G(\alpha, \lambda)$ is continuous on the closed rectangle, positive if $\lambda$ is positive, and equals zero when $\lambda=0$.

Now $Y \subset\left[\alpha_{1}, \alpha_{2}\right] \times[0, \tilde{\lambda}]$ is that component of $S^{-1}\{0\}$ containing $\left(\alpha^{*}, 0\right)$, which by Lemma 3.2 contains more than the single point $\left(\alpha^{*}, 0\right)$. It follows that there exist points $\left(\alpha^{\prime}, \lambda^{\prime}\right)$ in $Y$ with $\lambda^{\prime}>0$ with $S\left(\alpha^{\prime}, \lambda^{\prime}\right)=0$ and $\theta\left(\alpha^{\prime}, \lambda^{\prime}\right)$ positive. Since $Y$ is a connected set and $\theta$ is continuous on $Y$ there will exist points $(\alpha, \lambda)$ in $Y$ with $\lambda>0$ and $S(\alpha, \lambda)=0, \theta(\alpha, \lambda) / 2 \pi$ a positive rational number. The resulting surface $\mathbf{x}(u, v, \alpha, \lambda)$ is a closed 
surface. We have shown

MAIN THEOREM. There exists $(\alpha, \lambda)$ with $0<\alpha<\pi / 4$ and $\lambda>0$ so that the surface $\mathbf{x}(u, v, \alpha, \lambda): R^{2} \rightarrow R^{3}$ is a doubly periodic immersion with constant mean curvature $H=1 / 2$. This determines an immersion of a torus $T^{2}$ into $R^{3}$ with constant mean curvature. Furthermore, if $(\alpha, \lambda)$ are chosen so that $S(\alpha, \lambda)=0$ and $\theta(\alpha, \lambda)=\pi / n$ where $n$ is a positive integer, then the corresponding immersion will contain $2 n$ rectangular zones of positive Gaussian curvature and $2 n$ zones of negative Gaussian curvature.

IV. The differential equation. We now proceed with a discussion of Theorem 3.1 on existence of solutions to (3.3). The proof is based on the method used by V. Weston [18] on solutions to the P.D.E. $\Delta \omega+\lambda e^{\omega}=0$ on a region $\Omega$ with $\omega=0$ on $\partial \Omega$. This method was subsequently extended by $J$. Moseley $[12,13]$ to include differential equations of the form $\Delta \omega+\lambda\left(e^{\omega}+\sigma e^{-\omega}\right)=0$ on $\Omega$ with Dirichlet boundary data. Here $\sigma$ is an analytic function on $\Omega$. So if we set $\sigma=-1$ it is seen that the results of Weston and Moseley should apply to our situation where $\Omega$ is the rectangle $\Omega(\alpha)$. In their papers Weston and Moseley impose certain conditions on the domain $\Omega$ to insure that their procedure works. Except for the condition that its boundary be smooth, these conditions are met by rectangular domains. Their method does carry over to this case, as we shall show. However, some alterations in the argument are necessary and for this reason we shall carry through the proof in some detail.

In this section we shall change notation slightly, keeping it in agreement with the papers of Weston and Moseley. We let $D$ be the unit disk in the $z$-plane where $z=x_{1}+i x_{2}, x=\left(x_{1}, x_{2}\right) . \Omega$ is a domain in the $w=y_{1}+i y_{2}$ plane with $y=\left(y_{1}, y_{2}\right)$.

We let $w=f(z)$ be a conformal map of $D$ onto $\Omega$ so that for us $f(z) \equiv f(z, \alpha)$ will be the Schwartz-Christoffel map (3.1) of $D$ onto the rectangle $\Omega(\alpha) . f(z, \alpha)$ is continuous on $\bar{D} \times(0, \pi / 2)$ and is analytic in $z$ for $z \in D$. The inverse map $z=g(w, \alpha): \Omega(\alpha) \rightarrow D$ has a meromorphic extension to the entire $w$-plane which is analytic in a neighborhood of $\Omega(\alpha)$. As noted earlier, for each $\alpha, f(z, \alpha)$ is a Lipschitz (Hölder continuous) map of class $\operatorname{Lip}(1 / 2, \bar{D})$ with Lipschitz constant depending continuously on $\alpha$.

Let $\tilde{u}(y)$ be a solution to $\Delta \tilde{u}+\lambda h(\tilde{u})=0$ on $\Omega$. Then it follows that $u(x)=\tilde{u} \circ f(x)$ is a solution to $\Delta u+\lambda\left|f^{\prime}(z)\right|^{2} h(u)=0$ on $D$. We may convert these P.D.E.'s to integral equation form by using the Green's kernel for the Laplace operator.

$$
\tilde{u}\left(y_{0}\right)=\lambda \int_{\Omega} \tilde{k}\left(y, y_{0}\right) h[\tilde{u}(y)] d y
$$


where $\tilde{k}\left(y, y_{0}\right)$ is the Green's kernel for the Laplace operator on $\Omega$ with zero boundary data. Similarly, the transformed function $u(x)=\tilde{u}[f(x)]$ satisfies

$$
u\left(x_{0}\right)=\lambda \int_{D} k\left(x, x_{0}\right)\left|f^{\prime}(z)\right|^{2} h[u(x)] d x
$$

where $k\left(x, x_{0}\right)=(-1 / 2 \pi) \ln \left(\left|z-z_{0}\right| /\left|1-\bar{z}_{0} z\right|\right)$.

If $w=f(z)$ is the Schwartz-Christoffel map of $D$ onto $\Omega(\alpha)$, [we shall often suppress the dependence on $\alpha$ ] then a straightforward calculation reveals the following

(a) $f(z, \alpha)$ is odd in $z$

(b) $f(z, \alpha)$ is real for $z$ real, $\overline{f(z, \alpha)}=f(\bar{z}, \alpha)$.

(c) $f(z, \alpha)=z+2(\cos 2 \alpha) z^{3}+\cdots$

so that $f(0, \alpha)=0, f^{\prime}(0, \alpha)=1, f^{\prime \prime}(0, \alpha)=0,\left|f^{\prime \prime \prime}(0, \alpha)\right|<2$.

TheOREM 4.1. (In large part, due to Weston and Moseley.) There exists an open set 0 in the $(\alpha, \lambda)$ plane and a continuous map $(\alpha, \lambda) \rightarrow u(x, \alpha, \lambda)$ from 0 into $C(\bar{D})$, the space of continuous functions on $\bar{D}$ with the uniform norm, such that

(a) For each choice of $0<\alpha_{1}<\alpha_{2}<\pi / 2$ there exists $\tilde{\lambda}=\tilde{\lambda}\left(\alpha_{1}, \alpha_{2}\right)>$ 0 with $\left[\alpha_{1}, \alpha_{2}\right] \times(0, \tilde{\lambda}] \subset 0$.

(b) $u(x, \alpha, \lambda)=0$ for $x \in \partial D$

(c) $u(x, \alpha, \lambda)$ is a solution of the operator equation

$$
u\left(x_{0}, \alpha, \lambda\right)=\lambda \int_{D} k\left(x, x_{0}\right)\left|f^{\prime}(z, \alpha)\right|^{2}\left(e^{u}-e^{-u}\right) d x \equiv K[u]\left(x_{0}\right) .
$$

(Following the notation of Weston and Moseley, we shall write the right hand side of (4.4) as $K[u]\left(x_{0}\right) \equiv K_{\lambda}[u]\left(x_{0}\right)$ or simply $K(u)$.)

(d) On any subdomain of $\bar{D}$ which excludes a neighborhood of $z=0$ $u(x, \alpha, \lambda)$ converges to $4 \ln (1 /|z|)$ as $\lambda \rightarrow 0$.

Proof. The proof is in two parts. In Part I we construct an approximate solution which behaves in the correct asymptotic manner as $\lambda \rightarrow 0$. In Part II we use a modified Newton iteration procedure on the approximate solution to obtain a sequence converging to the exact solution.

Step 1. Construction of the approximate solution.

We start with a well-known result of Liouville that a function $\tilde{u}(w)$ defined by

$$
\lambda e^{\tilde{u}} \equiv 8\left|F^{\prime}(w)\right|^{2} /\left(1+|F(w)|^{2}\right)^{2}
$$


where $F(w)$ is a complex analytic function with at most simple zeros and poles is a solution to $\Delta \tilde{u}+\lambda e^{\tilde{u}}=0$. It is clear that $F(w)$ can have at most simple zeros. That $F(w)$ can have at most simple poles follows from the identity $\left|\Psi^{\prime}(w)\right| /\left(1+|\Psi(w)|^{2}\right)=\left|F^{\prime}(w)\right| /\left(1+|F(w)|^{2}\right)$ where $\Psi(w)=$ $1 / F(w)$. Therefore, if $w=f(z)$ and if we set $u(x)=\tilde{u} \circ f(z)$ where $z=$ $x_{1}+i x_{2}, x=\left(x_{1}, x_{2}\right), w=y_{1}+i y_{2}, y=\left(y_{1}, y_{2}\right)$ then we have

$$
\lambda e^{u(x)}=8\left|f^{\prime}(z)\right|^{-2}\left|F^{\prime}(z)\right|^{2} /\left(1+|F(z)|^{2}\right)^{2}
$$

is a solution to $\Delta u+\lambda\left|f^{\prime}(z)\right|^{2} e^{u}=0$ on $D$, where $F(z)$ is an analytic function on $D$ with at most simple zeros and poles. We rewrite (4.6) in the form

$$
e^{-u / 2}=(\lambda / 8)^{1 / 2}\left|f^{\prime}(z)\right|\left(1+|F(z)|^{2}\right) /\left|F^{\prime}(z)\right| \quad \text { [Weston]. }
$$

A nice reduction due to Moseley is the following [12, p. 937]. We set

$$
1 / F(z)=(\lambda / 8)^{1 / 2} \int^{z}\left[f^{\prime}(t) / v^{2}(t)\right] d t
$$

where $v(z)$ is an analytic function to be chosen so that the right hand side is single valued, Equation (4.7) now becomes

$$
e^{-u / 2}=|v(z)|^{2}+\frac{\lambda}{8}\left|v(z) \int^{z} \frac{f^{\prime}(t)}{v^{2}(t)} d t\right|^{2} .
$$

Next we write $v(z, \lambda)=z / G(z, \lambda)$ where $G(z, \lambda)=1+\lambda G_{1}(z)+$ $\cdots+\lambda^{n} G_{n}(z)$. The functions $G_{k}(z)$ are to be complex analytic on $D$. We obtain

$$
e^{-u / 2}=\frac{|z|^{2}+(\lambda / 8)\left|z \int^{z}\left[G^{2}(t, \lambda) f^{\prime}(t) / t^{2}\right] d t\right|^{2}}{|G(z, \lambda)|^{2}} .
$$

We intend to choose $G(z, \lambda)$ to be analytic in $D$ and continuous on $\bar{D}$. Furthermore since $f(z) \equiv f(z, \alpha)$ in an old analytic function of $z$ satisfying the conditions (4.3), it is necessary that $G_{z}(0, \lambda)=0$ if the integrated term is to be single valued. This will be the case if $G(z, \lambda)$ is even in $z$. We shall require

$$
\begin{aligned}
& \text { (a) } \overline{G(-z, \lambda)}=G(z, \lambda) \\
& \text { (b) } \overline{G(z, \lambda)}=G(\bar{z}, \lambda) .
\end{aligned}
$$

If $G(z, \lambda)$ does satisfy these conditions then $u(x)$ will be a function on $\bar{D}$ satisfying the symmetry condition

(a) $u(-z, \lambda)=u(z, \lambda)$

(b) $u(\bar{z}, \lambda)=u(z, \lambda)$. 
The function $u_{e}(x, \lambda) \equiv u(x, \lambda)$ satisfying (4.10) is an exact solution to the exponential D.E. $\Delta u+\lambda\left|f^{\prime}(z)\right|^{2} e^{u}=0$. We now search for an approximate solution to $\Delta u+\lambda\left|f^{\prime}(z)\right|^{2}\left(e^{u}-e^{-u}\right)=0$ by setting

$$
u(x, \lambda)=u_{e}(x, \lambda)+H(x, \lambda) \quad \text { (Moseley) }
$$

where $u_{e}(x, \lambda)$ is of the form (4.10) and $H(x, \lambda)=\lambda H_{1}(x)+\lambda^{2} H_{2}(x)+$ $\cdots+\lambda^{n} H_{n}(x)$. In order to obtain a sufficiently accurate starting solution we shall need to find an approximate solution of order 3 .

Note. This disagrees with Moseley [12] where an approximate starting solution of order 2 is deemed sufficient. However, there seems to be a miscalculation at one point causing the discrepancy. Order at least 3 seems necessary. As we shall see the method breaks down for order greater than 3, so our situation is fortuitous.

We establish the following.

LEMma 4.1. There exist functions $G_{i}(z) i=1,2,3$ which are complex analytic on $D$, continuous on $\bar{D}$ and which are symmetric (i.e. satisfy (4.11)). There exist real-valued functions $H_{\imath}(z) \in C^{2}(D) \cap C^{0}(\bar{D})$ satisfying the symmetry conditions (4.12), so that the following are true. If we set $G(z, \lambda)$ $=1+\lambda G_{1}(z)+\lambda^{2} G_{2}(z)+\lambda^{3} G_{3}(z)$ and $H(z, \lambda)=\lambda H_{1}(z)+\lambda^{2} H_{2}(z)+$ $\lambda^{3} \mathrm{H}_{3}(z)$ and define

$$
u_{0}(z, \lambda)=u_{e}(z, \lambda)+H(z, \lambda)
$$

where $u_{e}(z, \lambda)$ is given by (4.10), then $u_{0}(z, \lambda)$ will satisfy the conditions

I. $u_{0}(z, \lambda) \in C^{2}(D) \cap C^{0}(\bar{D})$ and is symmetric satisfying (4.12).

II. $\left|u_{0}(z, \lambda)\right| \in 0\left(\lambda^{4}\right)$ on $|z|=1$ as $\lambda \rightarrow 0$ (i.e. there exists $M$ with $\left|u_{0}(z, \lambda)\right| \leq M \lambda^{4}$ for $|z|=1$ and $\left.0<\lambda \leq \tilde{\lambda}\right)$.

III. If we set $K(u)$ to be the integral operator (4.4) then $\left\|u_{0}-K\left(u_{0}\right)\right\|$ $\in O\left(\lambda^{3} \ln (1 / \lambda)\right)$ where $\|v\|=\sup |v(z)|$ for $z \in \bar{D}$.

IV. As $\lambda \rightarrow 0$ the function $e^{-u_{0}(z, \lambda) / 2} \Rightarrow|z|^{2}$ uniformly on $\bar{D}$ (hence $u_{0}(z, \lambda) \Rightarrow 4 \ln (1 /|z|)$ uniformly on subregions of $\bar{D}$ which exclude a neighborhood of $z=0$.)

The bounds in II and III will depend continuously on $\alpha$.

Proof of Lemma. We need six equations in order to determine $G_{i}(z)$ and $H_{\imath}(z)$. Following the procedure of Weston [18] and Moseley we write

$$
N \equiv\left|z \int^{z} \frac{G^{2}(t, \lambda) f^{\prime}(t)}{t^{2}} d t\right|^{2} \text { and } K=|G(z, \lambda)|^{2}
$$

so that $e^{-u_{e} / 2}=\left(|z|^{2}+(\lambda / 8) N\right) / K$. Now set

(4.15) $E(z, \lambda)=\left[\Delta u+\lambda\left|f^{\prime}(z)\right|^{2}\left(e^{u}-e^{-u}\right)\right] K^{2}\left[|z|^{2}+(\lambda / 8) N\right]^{2}$. 
A direct calculation gives us

$$
\begin{aligned}
E(z, \lambda)= & (\Delta H) K^{2}\left(|z|^{2}+(\lambda / 8) N\right)^{2} \\
& +\lambda\left|f^{\prime}(z)\right|^{2}\left[K^{4}\left(e^{H}-1\right)-\left(|z|^{2}+(\lambda / 8) N\right)^{4} e^{-H}\right] .
\end{aligned}
$$

Our first requirement is to choose $G(z, \lambda)$ and $H(z, \lambda)$ in (4.16) so that $\partial^{k} E(z, \lambda) / \partial \lambda^{k}=0$ for $\lambda=0, z \in D$, and $k=1,2,3$. This gives us $E(z, \lambda) \in O\left(\lambda^{4}\right)$ for each $z \in D$. Our second requirement is that $|u(z, \lambda)|$ $\in O\left(\lambda^{4}\right)$ on $|z|=1$. The calculations are tedious but feasible. We sketch the work here.

$$
\begin{aligned}
& \Delta H= \lambda \Delta H_{1}+\lambda^{2} \Delta H_{2}+\lambda^{3} \Delta H_{3} \\
& K=|G|^{2}=1+\lambda\left(G_{1}+\overline{G_{1}}\right)+\lambda^{2}\left[\left(G_{2}+\overline{G_{2}}\right)+\left|G_{1}\right|^{2}\right] \\
&+\lambda^{3}\left[\left(G_{3}+\bar{G}_{3}\right)+G_{1} \bar{G}_{2}+\bar{G}_{1} G_{2}\right]+\cdots \\
& K^{2}= 1+\lambda\left[2\left(G_{1}+\overline{G_{1}}\right)\right]+Q \lambda^{2}+\cdots \\
& \quad \text { where } Q=2\left(G_{2}+\overline{G_{2}}\right)+2\left|G_{1}\right|^{2}+\left(G_{1}+\overline{G_{1}}\right)^{2} \\
& K^{4}= 1+\lambda\left[4\left(G_{1}+\bar{G}_{1}\right)\right]+\lambda^{2}\left[2 Q+4\left(G_{1}+\bar{G}_{1}\right)^{2}\right]+\cdots \\
& e^{H}-1= \lambda H_{1}+\lambda^{2}\left[H_{2}+\left(H_{1}^{2} / 2\right)\right] \\
&+\lambda^{3}\left[H_{3}+H_{1} H_{2}+\left(H_{1}^{3} / 6\right)\right]+\cdots \\
& e^{-H}= 1-\lambda H_{1}+\lambda^{2}\left[-H_{2}+\left(H_{1}^{2} / 2\right)\right] \\
&+\lambda^{2}\left[-H_{3}+H_{1} H_{2}-\left(H_{1}^{3} / 6\right)\right]+\cdots
\end{aligned}
$$

(4.17) $\int^{z} \frac{G^{2}(t, \lambda) f^{\prime}(t)}{t^{2}} d t$

$$
\begin{aligned}
& =\int^{z}\left[1+2 \lambda G_{1}+\lambda^{2}\left(2 G_{2}+G_{1}^{2}\right)+\cdots\right] \frac{f^{\prime}(t)}{t^{2}} d t \\
& \equiv\left[\frac{P_{0}(z)}{z}+c_{0}\right]+\lambda\left[\frac{P_{1}(z)}{z}+c_{1}\right]+\lambda^{2}\left[\frac{P_{2}(z)}{z}+c_{2}\right]+\cdots
\end{aligned}
$$

where

$$
\begin{aligned}
& P_{0}(z)=-f^{\prime}(0)+z \int_{0}^{z} \frac{f^{\prime}(t)-f^{\prime}(0)}{t^{2}} d t \\
& P_{i}(z)=-\Psi_{\imath}(0) f^{\prime}(0)+z \int_{0}^{z} \frac{\Psi_{i}(t) f^{\prime}(t)-\Psi_{\imath}(0) f^{\prime}(0)}{t^{2}} d t
\end{aligned}
$$

where $\Psi_{1}(z)=2 G_{1}(z), \Psi_{2}(z)=2 G_{2}(z)+G_{1}^{2}(z)$, etc. 
The functions $P_{i}(z)$ will be single-valued if $\Psi_{l}^{\prime}(0)=0$ which will be the case if $G_{i}^{\prime}(0)=0$. We may set the constants $c_{K}=0$, to obtain

$$
\begin{aligned}
& \begin{aligned}
N= & \left|z \int^{z} \frac{G^{2} f^{\prime}(t)}{t^{2}} d t\right|^{2}=\left|P_{0}\right|^{2}+\lambda\left(P_{0} \bar{P}_{1}+\bar{P}_{0} P_{1}\right) \\
& +\lambda^{2}\left[\left(P_{0} \bar{P}_{2}+\bar{P}_{0} P_{2}\right)+\left|P_{1}\right|\right]^{2}+\cdots
\end{aligned} \\
& {\left[|z|^{2}+(\lambda / 8) N\right]^{2}} \\
& =|z|^{4}+\left(|z|^{2}\left|P_{0}\right|^{2} / 4\right) \lambda \\
& \quad+\left[\left(|z|^{2}\left(P_{0} \bar{P}_{1}+\bar{P}_{0} P_{1}\right) / 4\right)+\left(\left|P_{0}\right|^{4} / 64\right)\right] \lambda^{2}+\cdots
\end{aligned}
$$

(b)

We substitute these expansions into (4.16), compute the coefficients of $\lambda$, $\lambda^{2}, \lambda^{3}$ in the expression for $E(z, \lambda)$, and set them equal to zero.

We also want $|u(z, \lambda)| \in O\left(\lambda^{4}\right)$ for $|z|=1$. This is equivalent to $e^{-u_{e} / 2}=e^{H / 2}$ on $|z|=1$ to order 3 . But $e^{-u_{e} / 2}=\left[|z|^{2}+(\lambda / 8) N\right] / K$ where $K=|G|^{2}$ by (4.10). We obtain the condition

$$
|G|^{2}=e^{-H / 2}\left[|z|^{2}+(\lambda / 8) N\right]
$$

to order 3 on $|z|=1$. We now have a total of six conditions. They are

$$
\begin{gathered}
\text { (I) } \Delta H_{1}-\left|f^{\prime}(z)\right|^{2}|z|^{4}=0 \text { on } D \\
\text { (II) } G_{1}+\bar{G}_{1}=\left|P_{0}\right|^{2} / 8-H_{1} / 2 \text { on }|z|=1 \\
\text { (III) } \Delta H_{2}+\left|f^{\prime}\right|^{2}\left\{H_{1} /|z|^{4}+\left[2|z|^{4}\left(G_{1}+\bar{G}_{1}\right)-|z|^{4}\left|P_{0}\right|^{2} / 4\right.\right. \\
\left.\left.\quad+|z|^{4} H_{1}\right]\right\}=0 \text { on } D \\
\text { (IV) }\left(G_{2}+\bar{G}_{2}\right)+\left|G_{1}\right|^{2}=\left(P_{0} \bar{P}_{1}+\bar{P}_{0} P_{1}\right) / 8-\left|P_{0}\right|^{2} H_{1} / 16 \\
\quad-H_{2} / 2+H_{1}^{2} / 8 \text { on }|z|=1 \\
\text { (V) }|z|^{4}\left(\Delta H_{3}\right)+\left(2|z|^{4}\left(G_{1}+\bar{G}_{1}\right)+|z|^{2}\left|P_{0}\right|^{2} / 4\right)\left(\Delta H_{2}\right) \\
+\left[|z|^{4} Q+\left(|z|^{2}\left|P_{0}\right|^{2}\left(G_{1}+\bar{G}_{1}\right) / 2\right)\right. \\
\left.+|z|^{2}\left(P_{0} \bar{P}_{1}+\bar{P}_{0} P_{1}\right) / 4+\left|P_{0}\right|^{4} / 64\right]\left(\Delta H_{1}\right) \\
+\left|f^{\prime}(z)\right|^{2}\left[4 H_{1}\left(G_{1}+\bar{G}_{1}\right)+H_{2}+H_{1}^{2} / 2\right] \\
\quad-\left|f^{\prime}(z)\right|^{2}(J)=0 \text { on } D
\end{gathered}
$$


where

$$
\begin{aligned}
& Q=2\left(G_{2}+\bar{G}_{2}\right)+2\left|G_{1}\right|^{2}+\left(G_{1}+\bar{G}_{1}\right)^{2} \text { and } \\
& J=|z|^{6}\left(P_{0} \bar{P}_{1}+\bar{P}_{0} P_{1}\right) / 2+3|z|^{4}\left|P_{0}\right|^{4} / 32-|z|^{6} H_{1}\left|P_{0}\right|^{2} / 2 \\
& -|z|^{8} H_{2}+|z|^{8} H_{1}^{2} / 2 \\
& \text { (VI) } \quad\left(G_{3}+\bar{G}_{3}\right)+\left(G_{1} \bar{G}_{2}+\bar{G}_{1} G_{2}\right) \\
& =\left(P_{0} \bar{P}_{2}+\bar{P}_{0} P_{2}\right) / 8+\left|P_{1}\right|^{2} / 8-H_{1}\left(P_{0} \bar{P}_{1}+\bar{P}_{0} P_{1}\right) / 16 \\
& +\left(\left|P_{0}\right|^{2} / 8\right)\left[-H_{1} / 2+H_{1}^{2} / 8\right] \\
& -\left[H_{3} / 2-H_{1} H_{2} / 4+H_{1}^{3} / 48\right] \text { on }|z|=1 .
\end{aligned}
$$

To find sufficiently smooth solution of this set of equations we shall use the following "well-known" theorems both of which may be found in Courant-Hilbert, Vol. II [2].

Privalov's Theorem [2, p. 401]. Let $F(z)=U(z)+i V(z)$ be an analytic function on the unit disk. Suppose that $U(z)$ is continuous on $\bar{D}$ and that on $\partial D, U(z)$ is a Lipschitz (Hölder) continuous function with exponent $\mu, 0<\mu<1$. (i.e. $U(z) \in \operatorname{Lip}(\mu, \partial D)$. There exists a constant $M$ with $\left|U\left(z_{1}\right)-U\left(z_{2}\right)\right| \leq M\left|z_{1}-z_{2}\right|^{\mu}$ for $\left.z_{1}, z_{2} \in \partial D\right)$. Then $F(z)$ itself is Lipschitz (Hölder) continuous on $\bar{D}$ with exponent $\mu$ (i.e. $F(z) \in \operatorname{Lip}(\mu, \bar{D})$ ).

Let $\Omega \subset R^{2}$ be a bounded domain with piecewise smooth boundary $\Gamma$ ( $\Omega$ is a rectangle). Let $0<\mu<1$ and $m \geq 0$ an integer be given. $\hat{C}^{m+\mu}(\Omega)$ in the Banach space of real-valued functions $u(x) \in C^{m}(\Omega) \cap C^{0}(\bar{\Omega})$ with norm $\|\hat{u}\|_{m+\mu}=\|\hat{u}\|_{m}+H_{\mu}\left[D^{m} u\right]$ where

$$
\|\hat{u}\|_{m}=\text { L.U.B. }\left[|u(p)|+d_{p}\left|D^{1} u(p)\right|+\cdots+d_{p}^{m}\left|D^{m} u(p)\right|\right]
$$

with the L.U.B. over all $p \in \Omega$ and over all partial derivatives of the indicated order, $d_{p}=$ distance $(p, \partial \Omega)$, and

$$
H_{\mu}\left[D^{m} u\right]=\text { L.U.B. } d_{p, q}^{m+\mu} \cdot \frac{\left|D^{m} u(p)-D^{m} u(q)\right|}{|p-q|^{\mu}}
$$

over all $p, q \in \Omega$ and $d_{p, q}=\min \left(d_{p}, d_{q}\right)$.

SCHAUder's THEOREM [2, p. 339]. Let $f \in \hat{C}^{\mu}(\Omega)$. There is a unique solution $u(x) \in \hat{C}^{2+\mu}(\Omega)$ to the system $\Delta u=f$ and $u=0$ on $\partial \Omega$. 
We solve the equations (4.20) $\mathrm{I}-\mathrm{VI}$ in succession to obtain functions $G_{i}(z), H_{i}(z), i=1,2,3$, so that the following are also true.

$H_{l}(z)$ and $G_{l}(z)$ are "symmetric". That is $G_{l}(z)$ satisfies condition (4.11) and $H_{l}(z)$ satisfies (4.12).

(b) $\quad G_{l}(z)$ is analytic on $D$ and $G_{l}(z)$ is continuous on $\bar{D}$ with $G_{l}(z) \in \operatorname{Lip}(1 / 2, \bar{D})$

(c) $H_{l}(z)=A_{l}[f(z, \alpha)]+p_{l}(z)$ where $p_{l}(z)$ is a harmonic polynomial and $A_{l}(w) \in C^{2+1 / 2}[\Omega(\alpha)] \subset C^{2}(\Omega(\alpha)) \cap C^{0}(\overline{\Omega(\alpha)})$ with $A_{l}(w)=0$ on $\partial \Omega(\alpha)$. Here $f(z, \alpha)$ is the Schwartz-Christoffel map of $D$ onto $\Omega(\alpha)$.

$$
H_{l}(z) \in O\left(|z|^{8-2 l}\right) \text { around } z=0 .
$$

Start with (4.20-I). Transform the D.E. for $H_{1}(z)$ to the domain $\Omega(\alpha)$ to obtain the D. E. $\Delta A_{1}(w)=|g(w)|^{4}=\Psi_{1}(w)$. Since $g(w)$ (the inverse of the Schwartz-Christoffel map $w=f(z))$ is analytic in a neighborhood of $\overline{\Omega(\alpha)}$, we have $\Psi_{1}(w) \in \hat{C}^{\mu}(\Omega(\alpha))$ for any $\mu, 0<\mu<1$. Thus there is a unique solution $A_{1}(w) \in C^{2+\mu}(\Omega(\alpha))$ to the system $\Delta A_{1}(w)=\Psi_{1}(w)$, $A_{1} \mid \partial \Omega=0$. Since $\Psi(w)$ satisfies the symmetry conditions, $A(w)$ does also. Now $A_{1}[f(z)]$ is a solution to the D.E. (4.20-I) and the right-bound side of this D. E., is of order $O(|z|)^{4}$ around $z=0$. Therefore, there is a harmonic polynomial $p_{1}(z)$ of degree 5 such that $H_{1}(z) \equiv A_{1}[f(z)]+p_{1}(z)$ is a solution (4.20-I) and $H_{1}(z) \in O\left(|z|^{6}\right)$ around $z=0$ (see [12, p. 945] for proof of existence of $p(z)$ ).

(4.20-II). Since $f(z) \in \operatorname{Lip}(1 / 2, \bar{D})$ it follows that $P_{0}(z)$ (see (4.17)) $\in \operatorname{Lip}(1 / 2, \bar{D})$ as well. Therefore the right-side of $(4.20-\mathrm{II})$ is of class $\operatorname{Lip}(1 / 2, \partial D)$. We may now apply Privalov's Theorem to claim the existence of an analytic function $G_{1}(z)$ satisfying (4.20-II) with $G_{1}(z) \in$ $\operatorname{Lip}(1 / 2, \bar{D})$. Furthermore, since the conditions on $|z|=1$ are symmetric, the arbitrary additive constant may to chosen so that $G_{1}(z)$ is symmetric. In particular, $G_{1}^{\prime}(0)=0$.

(4.20-III). We have a P.D.E. for $H_{2}(z)$ where $H_{1}(z)$ is a solution to $(4.20-\mathrm{I})$ and $G_{1}(z)$ solves (4.20-II). We proceed as in Case I. Transform the D. E. to the domain $\Omega(\alpha)$ to obtain an equation $\Delta A_{2}(w)=\Psi_{2}(w)$ where $\Psi_{2}$ is the non-homogeneous part of the equation composed with 
$z=g(w)$ and the $f^{\prime}(z)$ term drops out. Since $H_{1}(z) \in O\left(|z|^{6}\right)$ around $z=0$ we see that $\Psi_{2}(w) \in O\left(|w|^{2}\right)$ around $w=0$. Furthermore, an inspection of each term in the expansion for $\Psi_{2}(w)$ reveals that $\Psi_{2}(w) \epsilon$ $\hat{C}^{1 / 2}(\Omega(\alpha)) \subset C^{0}(\overline{\Omega(\alpha)})$. Therefore, by the Schauder Theorem, there exists a solution of $\Delta A_{2}(w)=\Psi_{2}(w)$ on $\Omega(\alpha), A_{2}=0$ on $\partial \Omega(\alpha)$ where $A_{2}(w) \in \hat{C}^{2+1 / 2}(\Omega(\alpha))$. Also $A_{2}(w)$ will satisfy the desired symmetry conditions.

As in Case I, we have $A_{2}[f(z)]$ is a solution to (4.20-III) of class $C^{2}(D) \cap C^{0}(\bar{D})$ vanishing on $\partial D$, where the right side of the D.E. is of order $O\left(|z|^{2}\right)$ around $z=0$. Thus there is a harmonic polynomial $p_{2}(z)$ of degree 3 such that $H_{2}(z)=A_{2}[f(z)]+p_{2}(z)$ is a solution to (4.20-III), $H_{2}(z) \in O\left(|z|^{4}\right)$ around $z=0$, and $H_{2}(z)$ satisfies the symmetry conditions.

(4.20-IV). On $|z|=1, H_{1}$ and $H_{2}$ are smooth functions while $P_{0}(z)$, $P_{1}(z), G_{1}(z)$ are in $\operatorname{Lip}(1 / 2, \bar{D})$. We may apply Privalov's Theorem to obtain a function $G_{2}(z) \in \operatorname{Lip}(1 / 2, \bar{D})$ which is complex analytic in $D$ satisfying (4.20-IV), which satisfies the symmetry conditions (4.11), and also $G_{2}^{\prime}(0)=0$.

$(4.20-\mathrm{V})$. We proceed as in cases I and III. In the domain $\Omega(\alpha)$ we obtain a D.E. of the form $\Delta A_{3}(w)=\Psi_{3}(w)$. We claim that $\Psi_{3}(w) \in$ $\hat{C}^{1 / 2}(\Omega(\alpha))$, is symmetric in $\Omega(\alpha)$ with $\Psi_{3}(w) \in O\left(|w|^{0}\right)$ around $w=0$. An inspection of the (many) individual terms comprising $\Psi_{3}(w)$ reveals that this is the case. In particular note that $H_{2}(z) \in O\left(|z|^{2}\right), \Delta H_{1}(z) \in$ $O\left(|z|^{4}\right), H_{2}(z) \in O\left(|z|^{4}\right), H_{1}(z) \in O\left(|z|^{6}\right)$ so that when we divide by the coefficient $|z|^{4}$ of $\Delta H_{3}(z)$ we find that $\Psi_{3}(w) \in O(1)$ near $w=0$. We also find that $\Psi_{3}(w) \in \hat{C}^{1 / 2}(\Omega(\alpha))$ and is symmetric. Let $A_{3}(w)$ be the solution to $\Delta A_{3}(w)=\Psi_{3}(w)$ with $A_{3}(w)=0$ on $\partial \Omega(\alpha)$ where $A_{3}(w) \epsilon$ $\hat{C}^{2+1 / 2}(\Omega)$. Now set $H_{3}(z)=A_{3}[f(z)]+p_{3}(z)$ where $p_{3}(z)$ is a harmonic polynomial of degree 1 chosen so that $H_{3}(z) \in O\left(|z|^{2}\right)$ around $z=0$. As in previous cases we find that $H_{3}(z) \in C^{2}(\Omega) \cap C^{0}(\bar{\Omega})$ is a symmetric solution to $(4.20-\mathrm{V})$ with smooth boundary values.

(4.20-VI). We have $G_{1}(z), G_{2}(z) \in \operatorname{Lip}(1 / 2, \bar{D})$ from which it follows that $P_{i}(z), i=1,2,3$, are in $\operatorname{Lip}(1 / 2, \bar{D})$ while $H_{i}(z) i=1,2,3$, are smooth functions on $|z|=1$. We can find a complex analytic function $G_{3}(z)$ satisfying $(4.20-\mathrm{VI})$ where $G_{3}(z)$ is symmetric and in class $\operatorname{Lip}(1 / 2, \bar{D})$.

We have constructed functions $G(z, \lambda), H(z, \lambda)$ and hence using (4.10) and (4.14) an approximate solution $u_{0}(z, \lambda)=u_{e}(z, \lambda)+H(z, \lambda)$. Because of the continuous dependence of the conformal map $w=f(z, \alpha)$ 
on $\alpha$ it follows that this approximate solution depends continuously on $\alpha$. To complete the proof of Lemma 4.1 it is necessary to verify condition III.

Going back to (4.15)-(4.16) we see that $E(z, \lambda)=\left|f^{\prime}(z)\right|^{2} e(z, \lambda)$ where $e(z, \lambda)$ is a continuous function on $\bar{D} \times[0, \bar{\lambda}]$ for some $\lambda>0$. Substitute this equation into (4.15) to obtain

$$
\Delta u_{0}+\lambda\left|f^{\prime}(z)\right|^{2}\left(e^{u_{0}}-e^{-u_{0}}\right)=m(z, \lambda)\left|f^{\prime}(z)\right|^{2} /\left(|z|^{2}+(\lambda / 8) N\right)^{2}
$$

where $m(z, \lambda)=e(z, \lambda) / K^{2}=e(z, \lambda) /|G|^{4}$ is a bounded function on $\bar{D}$, and $m(z, \lambda) \in O\left(\lambda^{4}\right)$ by our construction of $G(z, \lambda)$ and $H(z, \lambda)$. Let $v(z) \equiv v(z, \lambda)=K[u(z, \lambda)]$ where $K(u)$ is the integral operator (4.4) so that

$$
v\left(z_{0}\right)=\int_{D} k\left(z, z_{0}\right)\left[\lambda\left|f^{\prime}(z)\right|^{2}\left(e^{u_{0}}-e^{-u_{0}}\right)\right] d x .
$$

Then $\Delta v+\lambda\left|f^{\prime}(z)\right|^{2}\left(e^{u_{0}}-e^{-u_{0}}\right)=0$ on $D$ and $v=0$ on $\partial D$. Therefore $\Delta\left(u_{0}-v\right)=m(z, \lambda)\left|f^{\prime}(z)\right|^{2} /\left[|z|^{2}+(\lambda / 8) N\right]^{2}$ with $\left|u_{0}-v\right| \leq M \lambda^{4}$ on $\partial D$, and $|m(z, \lambda)| \leq M \lambda^{4}$ on $D$. Therefore

$$
\left\|u_{0}-K\left(u_{0}\right)\right\| \leq M \lambda^{4}+\sup _{z_{0} \in D} \int_{D} \frac{M \lambda^{4}\left|f^{\prime}(z)\right|^{2}}{\left[|z|^{2}+(\lambda / 8) N\right]^{2}} k\left(z, z_{0}\right) d x .
$$

We must find a bound on the integral. Our procedure follows that of Weston and Moseley. The only added difficulty in the singularity of $f^{\prime}(z)$ on the boundary of $D$. The correct bound for $\left\|u_{0}-K\left(u_{0}\right)\right\|$ and for (4.22) as asserted in Lemma 4.1 will be shown in the following lemma. Besides this bound, two other asymptotic bounds are needed to successfully apply the modified Newton method.

LEMMA 4.2. Let $u_{0}(z, \lambda)$ be the approximate solution constructed in Lemma 4.1. Let $K(u)$ be the integral operator (4.4) so that

$$
K_{u_{0}}^{\prime}(h)\left(x_{0}\right)=\lambda \int_{D} k\left(x, x_{0}\right)\left|f^{\prime}(z)\right|^{2}\left(e^{u_{0}}+e^{-u_{0}}\right) h(x) d x
$$

where $K(u)$ is considered as an operator on $C^{0}(\bar{D})$. The following estimates are true.

$$
\begin{aligned}
& \text { (a) }\left\|u_{0}-K\left(u_{0}\right)\right\| \in O\left(\lambda^{3} \ln (1 / \lambda)\right) \\
& \text { (b) }\left\|K_{u_{0}}^{\prime}\right\| \in O(\ln (1 / \lambda)) \\
& \text { (c) }\left\|\left(I-K_{u_{0}}^{\prime}\right)^{-1}\right\| \in O(1 / \lambda) \text { as } \lambda \rightarrow 0 .
\end{aligned}
$$

Proof. The determination of these estimates were carried out originally by Weston [18] for the $e^{u}$ case and extended by Moseley $[12,13]$ for the more general differential equation. For us, the added difficulty is in 
the singularity of $f^{\prime}(z)$ on $\partial D$, which as we shall see is a removable problem. Moreover, the symmetry of the conformal map $f(z)$ makes some of the technical estimates a bit easier. For completeness, we shall sketch the derivation of the estimates here. For some details one should refer to the paper of Weston [18]. The bounds (a) and (b) are straightforward while (c) is tricky.

We first obtain an asymptotic expression in $\lambda$ for the kernel $\lambda\left|f^{\prime}(z)\right| e^{u_{0}}$ valid in a neighborhood of $z=0$ in $D$. Now $f(z)=z+a_{3} z^{3}+a_{5} z^{5}+$ $\cdots$ around $z=0$. A straightforward calculation gives

$$
\left|f^{\prime}(z)\right|^{2}=1+p r^{2} \cos 2 \theta+r^{4} m_{1}(z)
$$

where $p=f^{\prime \prime \prime}(0)=6 a_{3}, z=r e^{i \theta}$ and $m_{1}(z)$ is a function bounded in any disk $D_{a}=\{z|| z \mid \leq a\}$ where $a<1$. As noted in (4.3) $|p|<2$ when $f(z)$ is the Schwartz-Christoffel map. From (4.10) and (4.14) and Step 1, we have

$$
e^{u_{0}}=e^{u_{e}} e^{H}=\left[|G|^{4} /\left(|z|^{2}+(\lambda / 8) N\right)^{2}\right] e^{H} \text { on } \bar{D} .
$$

Now take the expansion for $N$ as defined by (4.18), make use of the expansions

$$
\begin{aligned}
& \left|P_{0}\right|^{2}=1-p r^{2} \cos 2 \theta+r^{4} m_{2}(z) \\
& P_{0} \bar{P}_{1}+\bar{P}_{0} P_{1}=4 G(0)+O\left(|z|^{2}\right)=4 s+r^{2} m_{3}(z)
\end{aligned}
$$

where $m_{2}(z), m_{3}(z)$ are bounded functions on $\bar{D}$. For convenience we set $\lambda / 8 \equiv \mu$, and find

$$
\begin{gathered}
{\left[|z|^{2}+(\lambda / 8) N\right]=r^{2}+\mu N=\left(r^{2}+\mu\right)(1-T)} \\
T=\frac{\mu p r^{2} \cos 2 \theta}{r^{2}+\mu}-\frac{32 \mu^{2} s}{r^{2}+\mu}+\frac{\mu r^{4} m_{2}(z)}{r^{2}+\mu}+\frac{8 \mu^{2} r^{2} m_{3}(z)}{r^{2}+\mu} \\
+\frac{\mu^{4} \Gamma(z, \mu)}{r^{2}+\mu}
\end{gathered}
$$

where $\Gamma(z, \mu)$ is bounded for $z \in \bar{D}$. We note in particular that $T(z, \lambda) \in$ $O(\lambda)$ on $\bar{D}$.

In similar fashion we have $|G|^{4}=1+2 \lambda\left(G_{1}+\bar{G}_{1}\right)+O\left(\lambda^{2}\right)$ on $\bar{D}$ so that $|G|^{4}=1+4 s \lambda+\lambda r^{2} m_{4}(z, \lambda)+\lambda^{2} m_{5}(z, \lambda)$ where $m_{4}, m_{5}$ are bounded functions for $z \in \bar{D}$ and $0 \leq \lambda \leq \tilde{\lambda}$.

$$
e^{H}=1+\left(\lambda H_{1}+\lambda^{2} H_{2}+\lambda^{2} H_{3}\right)+\cdots=1+\lambda r^{6} m_{6}+\lambda^{2} m_{7}
$$


where $m_{6}, m_{7}$ are bounded functions on $\bar{D} \times[0, \tilde{\lambda}]$. Putting these estimates together we find

$$
\begin{aligned}
\rho(z, \lambda) \equiv \lambda\left|f^{\prime}(z)\right|^{2} e^{u_{0}}=\lambda\left|f^{\prime}(z)\right|^{2} e^{u_{e}} e^{H} & \\
= & \frac{1}{\left(r^{2}+\mu\right)^{2}}\left[1+p r^{2} \cos 2 \theta+4 \lambda s+\frac{2 \mu p r^{2} \cos 2 \theta}{r^{2}+\mu}\right. \\
& \left.-\frac{64 \mu^{2} s}{r^{2}+\mu}+R(z, \lambda)\right]
\end{aligned}
$$

where $R(z, \lambda)$ may be written in the form

$$
R(z, \lambda)=r^{4} n_{1}(z, \lambda)+\lambda r^{2} n_{2}(z, \lambda)+\lambda^{2} n_{3}(z, \lambda) .
$$

Here $n_{\imath}(z, \lambda)$ are continuous functions which are uniformly bounded on any disk $D_{a}$ where $a<1$ and $0 \leq \lambda \leq \tilde{\lambda}$ (some positive $\tilde{\lambda}$ ). The only factor preventing a uniform bound on $D_{1}$ is the $\left|f^{\prime}(z)\right|^{2}$ term.

If we remove the $\left|f^{\prime}(z)\right|^{2}$ term from (4.27) then the following estimate holds on $\bar{D}$. In particular we write

$$
\frac{1}{\left(|z|^{2}+\mu N\right)^{2}}=\frac{1}{\left(r^{2}+\mu\right)^{2}}\left[1+2 T+\frac{T^{2}(3-2 T)}{(1-T)^{2}}\right]
$$

and since $T \in O(\lambda)$ on $\bar{D}$ we have $(3-2 T) /(1-T)^{2}$ is bounded on $\bar{D}$ for small $\lambda$. We thus find

$$
\lambda e^{u_{0}}=\frac{\lambda}{r^{2}+\mu}\left[1+4 \lambda s+\frac{2 \mu p r^{2} \cos 2 \theta}{r^{2}+\mu}-\frac{64 \mu^{2} s}{r^{2}+\mu}+\tilde{R}(z, \lambda)\right]
$$

with $\tilde{R}(z, \lambda)$ of the form (4.27b) except that the estimate is valid on the full disk $\bar{D}$.

We now show

$$
\sup _{z_{0} \in D} \int_{D} k\left(z, z_{0}\right) \frac{\lambda\left|f^{\prime}(z)\right|^{2}}{\left(|z|^{2}+(\lambda / 8) N\right)^{2}} d x \in O\left(\ln \left(\frac{1}{\lambda}\right)\right) .
$$

We make our estimate by splitting the integral over two regions of integration, $D_{a}=$ disk of radius $a<1$ center $z=0$, and $D_{a}^{\prime}=D_{1}-D_{a}$. Denote the integral over $D_{a}$ by $A$, and the integral over $D_{a}^{\prime}$ by $B$, so that $I=A+B$ where $I=I\left(z_{0}\right)$ is the integral (4.29).

$$
|B| \leq \frac{\lambda}{a^{4}} \int_{D} k\left(z, z_{0}\right)\left|f^{\prime}(z)\right|^{2} d x=\frac{\lambda}{a^{4}} \int_{\Omega(\alpha)} \tilde{k}\left(w, w_{0}\right) d w
$$


by a change of variables where $f(z)=w$ is the conformal map of $D$ onto $\Omega(\alpha)$, and $\tilde{k}\left(w, w_{0}\right)$ is Green's function for the Laplace operator with 0 boundary data on $\Omega(\alpha)$. Therefore $|B| \leq \lambda M(\alpha) / a^{4} \in O(\lambda)$, and $M(\alpha)$ is a constant depending continuously on $\alpha$. We estimate $|A|$.

$$
\begin{aligned}
|A| & =\int_{D_{a}} k\left(z, z_{0}\right) \frac{\lambda\left|f^{\prime}(z)\right|^{2}}{\left(|z|^{2}+(\lambda / 8) N\right)^{2}} d x \\
& \leq C \int_{D_{a}} k\left(z, z_{0}\right) \frac{\lambda}{\left(r^{2}+\mu\right)^{2}} d x \leq C \int_{D} k\left(z, z_{0}\right) \frac{\lambda}{r^{2}+\mu^{2}} d x
\end{aligned}
$$

where we have used the fact that $\left|f^{\prime}(z)\right|$ is bounded on $D_{a}$, and from (4.26) we see $|z|^{2}+(\lambda / 8) N>\left(r^{2}+\mu\right) / 2$ since $T \in O(\lambda)$ on $\bar{D}$. Now we make use of the following.

Special Calculation. Let $\rho_{0}(z)=8 \mu /\left(r^{2}+\mu\right)^{2}$. For any triple of non-negative integers $[i, j, k]$ let $C[i, j, k]$ be defined by

$$
C[i, j, k]=\sup _{z_{0} \in D} \int_{D} k\left(z, z_{0}\right) \rho_{0}(z) \frac{\mu^{i} r^{j}}{\left(r^{2}+\mu\right)^{k}} d x
$$

where $k\left(z, z_{0}\right)$ is Green's function for the Laplacian with zero boundary data on $\bar{D}$. We have the following estimates

$$
\begin{array}{lll}
C[0,0,0] \in O(\ln (1 / \lambda)) & C[0,0,1] \in O\left(\lambda^{-1} \ln (1 / \lambda)\right) \\
C[0,1,0] \in O\left(\lambda^{1 / 2} \ln (1 / \lambda)\right) & C[0,1,1] \in O\left(\lambda^{-1 / 2} \ln (1 / \lambda)\right) \\
C[0,2,0] \in O(\lambda \ln (1 / \lambda)) & C[0,0,2] \in O\left(\lambda^{-2} \ln (1 / \lambda)\right) . \\
C[0,2,1] \in O(\ln (1 / \lambda)) & &
\end{array}
$$

Proof. This notation is due to Moseley [13], who had a similar list except $k\left(z, z_{0}\right)$ was replaced by the singular part of $k\left(z, z_{0}\right)=\ln \left(\left|z-z_{0}\right|\right)$. The proof of these estimates is most easily obtained by solving the P.D.E. associated with the integral operator.

$$
\Delta v+\rho_{0}(z)\left[\mu^{i} r^{j} /\left(r^{2}+\mu\right)^{k}\right]=0 \text { on } D
$$

$v=0$ on $\partial D$. The solution is radially symmetric and the corresponding O.D.E. can be solved explicitly.

We now see that $|A| \in O(\ln (1 / \lambda))$ and from this the bound (4.29) follows.

Now return to the bound (4.22) for $\left\|u_{0}-K\left(u_{0}\right)\right\|$. Using (4.29) and substituting into (4.22) we find

$$
\left\|u_{0}-K\left(u_{0}\right)\right\| \leq M \lambda^{4}+O\left(\lambda^{3} \ln (1 / \lambda)\right) \in O\left(\lambda^{3} \ln (1 / \lambda)\right) .
$$

This proves estimate (4.23-a) and completes the proof of Lemma 4.1. 
We now verify estimate (4.23-b). We write $K_{u_{0}}^{\prime}(h)=K_{1, u_{0}}^{\prime}(h)+$ $K_{2, u_{0}}^{\prime}(h)$ as a sum where $K_{1, u_{0}}^{\prime}(h)$ has the $e^{u_{0}}$ term in the integrand and $K_{2, u_{0}}^{\prime}(h)$ contains the $e^{-u_{0}}$ term. We estimate $\left\|K_{2, u_{0}}^{\prime}\right\|$ first. Clearly

$$
\left\|K_{2, u_{0}}^{\prime}\right\| \leq \sup _{z_{0} \in D} \lambda \int_{D} k\left(z, z_{0}\right)\left|f^{\prime}(z)\right|^{2} e^{-u_{0}(z)} d x .
$$

But

$$
e^{-u_{0}}=e^{-u_{e}} e^{-H}=\left[\frac{|z|^{2}+(\lambda / 8) N}{|G|^{4}}\right] e^{-H} \in O(1) \text { on } \bar{D}
$$

since $|G|$ is bounded away from 0 for small $\lambda$. Therefore

$$
\left\|K_{2, u_{0}}^{\prime}\right\| \leq \sup _{z_{0} \in D} \lambda C \int_{D} k\left(z, z_{0}\right)\left|f^{\prime}(z)\right|^{2} d x \in O(\lambda) .
$$

To estimate $\left\|K_{1, u_{0}}^{\prime}\right\|$ we note that

$$
e^{u_{0}}=\frac{|G|^{4}}{\left(|z|^{2}+(\lambda / 8) N\right)^{2}} e^{H} \leq \frac{C}{\left(|z|^{2}+(\lambda / 8) N\right)^{2}}
$$

and

$$
\left\|K_{1, u_{0}}^{\prime}\right\| \leq \sup _{z_{0} \in D} \lambda \int_{D} k\left(z, z_{0}\right)\left|f^{\prime}(z)\right|^{2} e^{u_{0}(z)} d x .
$$

Substitute the estimate for $e^{u_{0}}$ into the integral and use the estimate (4.29). We find $\left\|K_{1, u_{0}}^{\prime}\right\| \in O(\ln (1 / \lambda))$. Therefore $\left\|K_{u_{0}}^{\prime}\right\| \in O(\ln (1 / \lambda))$ and (4.23b) follows.

We must now show $\left\|\left(I-K_{u_{0}}^{\prime}\right)^{-1}\right\| \in O(1 / \lambda)$ as $\lambda \rightarrow 0$. We follow the method first used by Weston [18] and subsequently by Moseley [12]. Decompose the operator $K_{u_{0}}^{\prime}$ as follows. Write

$$
\begin{aligned}
K_{u_{0}}^{\prime}(h) & =K_{0}(h)+L(h) \text { where } \\
K_{0}(h) & =\int_{D} k\left(z, z_{0}\right) \rho_{0}(z) h(z) d x \\
\rho_{0}(r) & =\lambda /\left(r^{2}+\mu\right)^{2}=8 \mu /\left(r^{2}+\mu\right)^{2}, \quad \lambda=8 \mu .
\end{aligned}
$$

so that $K_{0}$ incorporates the "singular" part of the kernel of $K_{u_{0}}^{\prime}($ as $\lambda \rightarrow 0)$ and is an operator whose properties can be directly calculated.

Consider the eigenvalue problem $K_{0}(\varphi)=\Lambda \varphi$. This is equivalent to the differential equation

$$
\begin{gathered}
\Delta \varphi+(1 / \Lambda) \rho_{0}(x) \varphi=0 \text { on } D \\
\varphi=0 \text { on } \partial D .
\end{gathered}
$$


Since $\rho_{0}(z)=\rho_{0}(r)$ has radial symmetry, the eigenfunctions may be obtained by the method of separations of variables $\varphi(r, \theta)=\Psi(r) e^{\imath m \theta}$, giving us the O.D.E. for $\Psi(r)$ as

$$
\begin{aligned}
& \left(r \Psi_{r}\right)_{r} / r+\left[\rho_{0}(r) / \Lambda-m^{2} / r^{2}\right] \Psi(r)=0 \\
& \text { with } \Psi(0) \text { bounded, } \Psi(1)=0 .
\end{aligned}
$$

Now make the change of variables $\xi=\left(\mu-r^{2}\right) /\left(\mu+r^{2}\right)$ and (4.34) becomes

$$
\left[\left(1-\xi^{2}\right) \Psi_{\xi}\right]_{\xi}+\left[2 \sigma-m^{2} /\left(1-\xi^{2}\right)\right] \Psi=0
$$

where $\sigma=1 / \Lambda$ and the boundary conditions are $\Psi(1)$ bounded, $\Psi\left(\xi_{\mu}\right)=$ $0, \xi_{\mu}=(\mu-1) /(\mu+1)$. The solutions to (4.35) which are bounded at $\xi=1$ are the Legendre functions and associated Legendre functions (see Lebedev [11], for example). We have

$$
\begin{array}{ll}
m=0, & \Psi_{0}(\xi)=P_{\nu}(\xi) \quad \text { where } 2 \sigma=\nu(\nu+1) \\
m=1, & \Psi_{1}(\xi)=P_{\nu}^{1}(\xi) .
\end{array}
$$

The values of $\nu$ and hence $\Lambda$ are determined by the boundary condition $\Psi[(\mu-1) /(\mu+1)]=0$.

These calculations are essentially carried out by Weston [18] although his boundary conditions differ slightly. One uses an asymptotic estimate for $P_{\nu}^{m}(\xi)$ valid near $\xi=-1$ (as $\left.\mu \rightarrow 0\right)$ [18, page 1050]. One obtains the following asymptotic estimates for the largest eigenvalues with corresponding eigenfunctions.

\begin{tabular}{l|l|l|l|l}
$k$ & $\Lambda_{K}=2 /\left[\nu_{k}\left(\nu_{k}+1\right)\right]$ & \multicolumn{1}{|c|}{$\nu_{k}$} & $\Psi_{k}(\xi)$ & $\varphi_{k}(r, \theta)$ \\
\hline 1 & $2 \ln (1 / \mu)$ & {$[\ln (1 / \mu)]^{-1}$} & $P_{\nu_{0}}(\xi)$ & $\Psi_{1}(r)$ \\
\hline 2 & $1-3 \mu$ & $1+2 \mu$ & $P_{\nu_{1}}^{1}(\xi)$ & $\Psi_{2}(r) e^{\imath \theta}$ \\
\hline 3 & $1-3 \mu$ & $1+2 \mu$ & $P_{\nu_{1}}^{1}(\xi)$ & $\Psi_{2}(r) e^{-\imath \theta}$ \\
\hline 4 & $1-\frac{3}{2}[\ln (1 / \mu)]^{-1}$ & $1+[\ln (1 / \mu)]^{-1}$ & $P_{\nu_{4}}(\xi)$ & $\Psi_{4}(r)$ \\
\hline
\end{tabular}

The remaining eigenvalues are less than $(1 / 3)$ as $\lambda \rightarrow 0$ and so remain bounded away from one for small $\lambda$. The eigenfunctions are orthogonal with respect to the weight function $\rho_{0}(z)$ on the unit disk. We may also normalize them so that

$$
\left(\varphi_{\imath} \cdot \varphi_{j}\right)_{\rho_{0}}=\int_{D} \varphi_{i}(x) \bar{\varphi}_{J}(x) \rho_{0}(x) d x=\delta_{\imath \jmath} .
$$


Now let $P$ be the projection operator

$$
P(h)=\sum_{j=2}^{4} \Lambda_{j}\left(\varphi_{j} \cdot h\right) \varphi_{j}
$$

so that we may write from $(4.32)$

$$
K_{u_{0}}^{\prime}=P+\left(K_{0}-P\right)+L \equiv P+N+L .
$$

$N$ is a symmetric operator on $L^{2}(D)$ whose eigenvalues are bounded away from 1. It is compact as an operator either on the space $L^{2}(D)$ or $C^{0}(\bar{D})$. It follows from the Hilbert-Schmidt theory that $(I-N)^{-1}$ exists as a bounded operator on $C^{0}(\bar{D})$ for small $\lambda$ and its norm is uniformly bounded (i.e. $\left\|(I-N)^{-1}\right\| \in O(1)$ ) (see [18, p. 1045] and [5, Chap. 12]). Now write

$$
\left(I-K_{u_{0}}^{\prime}\right)(v)=w .
$$

We need to show $\|v\| \leq c(\lambda)\|w\|$ where $|c(\lambda)| \in O(1 / \lambda)$. Making use of the decomposition (4.38) and the fact that $(I-N)$ is invertible, one may rewrite (4.39) after a "few" manipulations in the form

$$
v=(I-M)^{-1}(I-N)^{-1} P v+(I-M)^{-1}(I-N)^{-1} w
$$

where $M=(I-N)^{-1} L$. However $N \varphi_{j}=\left(K_{0}-P\right) \varphi_{j}=0$ so that (4.41) reduces to

$$
v=(I-M)^{-1} P v+(I-M)^{-1}(I-N) w .
$$

Take the inner product (with weight function $\rho_{0}$ over $D$ ) of both sides of (4.42) with $\varphi_{i}$, set $c_{j}=\Lambda_{j}\left(v \cdot \varphi_{j}\right)$ and one obtains the linear system [18, p. 1045]

$$
\begin{gathered}
\sum_{j=2}^{4} a_{i j} c_{j}+w_{i}=0, \quad i=2,3,4, \\
a_{i j}=\left((I-M)^{-1} \varphi_{j} \cdot \varphi_{i}\right)_{\rho_{0}}-\delta_{i j} / \Lambda_{l} \\
w_{i}=\left((I-M)^{-1}(I-N)^{-1} w \cdot \varphi_{l}\right)_{\rho_{0}} .
\end{gathered}
$$

It is sufficient to show that $\left|c_{i}\right| \leq r(\lambda)\|w\|$ where $r(\lambda) \in O(1 / \lambda)$. To do this we need to estimate the size of the elements of the matrix $\left(a_{i j}\right)$. In the course of doing this we will also see that the operator $L$ (see 4.32) satisfies $\|L\| \in O(\lambda \ln (1 / \lambda))$ which will show that $(I-M)^{-1}$ where $M=$ $(I-N)^{-1} L$, is a uniformly bounded operator on $C^{0}(\bar{D})$. We recall (4.31) (4.32) and write $K_{u_{0}}^{\prime}=K_{0}+L$ and also write $(I-M)^{-1}=I+$ $M+M^{2}(I-M)^{-1}$ giving us

(4.44) $a_{i j}=\left(1-\Lambda_{l}^{-1}\right) \delta_{i j}+\left(L \varphi_{J} \cdot \varphi_{i}\right)_{\rho_{0}}+\left(M^{2}(I-M)^{-1} \varphi_{J} \cdot \varphi_{\imath}\right)_{\rho_{0}}$ 
where we have made use of the observation

$$
\left(M \varphi_{j} \cdot \varphi_{i}\right)=\left((I-N)^{-1} L \varphi_{j} \cdot \varphi_{i}\right)=\left(L \varphi_{j} \cdot \varphi_{i}\right) .
$$

Formula (4.36) for the asymptotic eigenvalues of the operator $K_{0}$ gives

$$
\begin{aligned}
& \left(1-\Lambda_{2}^{-1}\right)=-3 \mu+o(\mu)=\left(1-\Lambda_{3}^{-1}\right) \\
& \left(1-\Lambda_{4}^{-1}\right)=\frac{-3}{2}[\ln (1 / \mu)]^{-1}+o\left([\ln (1 / \mu)]^{-1}\right) .
\end{aligned}
$$

We now estimate the expression $\left(L \varphi_{j} \cdot \varphi_{i}\right)$. We write $L=\left(K_{1, u_{0}}^{\prime}-K_{0}\right)+$ $K_{2, u_{0}}^{\prime}$ and consider the operator $K_{2, u_{0}}^{\prime}$ first. The kernel for $K_{2, u_{0}}^{\prime}$ is $k\left(x, x_{0}\right)\left[\lambda\left|f^{\prime}(z)\right|^{2} e^{-u_{0}}\right]$. Making use of the formulae (4.25-27) we find

$$
\lambda\left|f^{\prime}(z)\right|^{2} e^{-u_{0}}=\lambda\left(r^{2}+\mu\right)^{2} m(z, \lambda)
$$

where $m(z, \lambda)$ is a continuous bounded function on any subdisk $D_{a}<D$ where $a<1$, while $\lambda\left|f^{\prime}(z)\right|^{2} e^{-u_{0}}=\lambda\left|f^{\prime}(z)\right|^{2} n(z, \lambda)$ on $\bar{D}$ where $n(z, \lambda)$ is uniformly bounded on $\bar{D}$ for $\lambda$ small.

We compute $\left(K_{2, u_{0}}^{\prime}\left(\varphi_{j}\right) \cdot \varphi_{i}\right)_{\rho_{0}}$ by reversing the order of integration in the iterated integral and noting that $\varphi_{l}$ is an eigenfunction for the operator $K_{0}$. We find

$$
\left(K_{2, u_{0}}^{\prime}\left(\varphi_{j}\right) \cdot \varphi_{i}\right)_{\rho_{0}}=\Lambda_{i}\left[\int_{D_{a}} \lambda\left(r^{2}+\mu\right)^{2} m \varphi_{j} \bar{\varphi}_{l} d x+\int_{D a} \lambda\left|f^{\prime}(z)\right|^{2} n \varphi_{j} \bar{\varphi}_{i} d x\right] .
$$

Following Weston [18], we estimate the first integral by making the change of variable $\xi=\left(\mu-r^{2}\right) /\left(\mu+r^{2}\right)$ used in (4.35). The eigenfunctions $\varphi_{i}(i=2,3,4)$ have asymptotic expressions

$$
\begin{aligned}
& \varphi_{2}(r, \theta) \rightarrow c P_{\nu_{1}}^{1}(\xi) e^{i \theta}=\Phi(\xi) e^{i \theta} \\
& \varphi_{3}(r, \theta) \rightarrow c P_{\nu_{1}}^{1}(\xi) e^{-i \theta}=\Phi(\xi) e^{-\imath \theta} \\
& \varphi_{4}(r, \theta) \rightarrow c^{\prime} P_{\nu_{4}}(\xi)=\Psi(\xi) .
\end{aligned}
$$

Since $\nu \rightarrow 1$ as $\lambda \rightarrow 0$ we may approximate the normalized eigenfunctions by

$$
\Phi(\xi)=\frac{1}{4}(3 / \pi)^{-1 / 2}\left(1-\xi^{2}\right)^{1 / 2}, \quad \Psi(\xi)=c \xi .
$$

For $i=2,3$ we have

$$
\begin{aligned}
& \int_{D_{a}} \lambda\left(r^{2}+\mu\right)^{2} m(z, \lambda)\left|\varphi_{j} \varphi_{i}\right| d x \leq M(a) \int_{0}^{1} \lambda\left(r^{2}+\mu\right)^{2}|\Phi(r)|^{2} d r \\
& \leq \lambda M(a) \int_{\xi_{\mu}}^{1} \frac{4 \mu^{2}}{(1+\xi)^{2}}\left(1-\xi^{2}\right) \frac{\mu}{(1+\xi)^{2}} d \xi \\
& \leq M(a) \lambda^{4} \int_{\xi_{\mu}}^{1}(1+\xi)^{-3} d \xi \leq M(a) \lambda^{2}
\end{aligned}
$$


where we have set $\left(r^{2}+\mu\right)=\mu[2 /(1+\xi)], r d r=\left[\mu /(1+\xi)^{2}\right] d \xi$ and $\xi_{\mu}=(\mu-1) /(\mu+1)$. Here $M(a)$ is a constant depending only on $a$.

Also

$$
\begin{aligned}
\int_{D_{a}^{\prime}}\left|f^{\prime}(z)\right|^{2} n\left|\varphi_{l} \varphi_{i}\right| d x & \leq \lambda \int_{D_{a}^{\prime}}\left|f^{\prime}(z)\right|^{2} M d x \\
& \leq \lambda M \int_{\Omega_{a}^{\prime}(\alpha)} d w=\varepsilon(a) \lambda \quad \text { where } \operatorname{limit}_{a \rightarrow 1} \varepsilon(a)=0 .
\end{aligned}
$$

We have shown

$$
\left|\left(K_{2, u_{0}}^{\prime}\left(\varphi_{J}\right) \cdot \varphi_{i}\right)_{\rho_{0}}\right| \leq M(a) \lambda^{2}+\varepsilon(a) \lambda, \quad i, j \in\{2,3\} .
$$

Similar calculations give

$$
\begin{aligned}
& \left|\left(K_{2, u_{0}}^{\prime} \varphi_{2} \cdot \varphi_{4}\right)_{\rho_{0}}\right| \leq M(a) \lambda^{3 / 2}+\varepsilon(a) \lambda \\
& \left|\left(K_{2, u_{0}}^{\prime} \varphi_{4} \cdot \varphi_{4}\right)_{\rho_{0}}\right| \leq M \lambda .
\end{aligned}
$$

It was shown earlier that $\left\|K_{2, u_{0}}^{\prime}\right\| \in O(\lambda)$. Thus

$$
\left(K_{2, u_{0}}^{\prime} \varphi_{j} \cdot \varphi_{l}\right)_{\rho_{0}}=\left(\begin{array}{c|c}
M(a) \lambda^{2} & M(a) \lambda^{3 / 2} \\
\hline M(a) \lambda^{3 / 2} & M(a) \lambda
\end{array}\right)+\lambda\left(\varepsilon_{l j}(a)\right) .
$$

Next we examine the operator $K_{1, u_{0}}^{\prime}-K_{0} \equiv L_{1}$. We use the representation (4.27) for the kernel of $K_{1, u_{0}}^{\prime}$.

$$
\begin{gathered}
\lambda\left|f^{\prime}(z)\right|^{2} e^{u_{0}}=\left[\lambda /\left(r^{2}+\mu\right)^{2}\right](1+\eta+R), \quad \eta=\eta_{1}+\eta_{2}, \\
\eta_{1}=p r^{2} \cos 2 \theta+\frac{2 \mu p r^{2} \cos 2 \theta}{r^{2}+\mu}=\frac{1+2 \mu}{r^{2}+\mu} p r^{2} \cos 2 \theta \\
\eta_{2}=4 \lambda s-\frac{64 \mu^{2} s}{r^{2}+\mu}=32 \mu s \frac{r^{2}-\mu}{r^{2}+\mu} \\
R(z, \lambda)=r^{4} n_{1}(z, \lambda)+\lambda r^{2} n_{2}(z, \lambda)+\lambda^{2} n_{3}(z, \lambda)
\end{gathered}
$$

where the $\eta_{t}(z, \lambda)$ are bounded on any $D_{a}, a<1$. On $D_{a}^{\prime}$ we have the estimate

$$
\lambda\left|f^{\prime}(z)\right|^{2} e^{u_{0}} \leq \lambda M\left|f^{\prime}(z)\right|^{2} .
$$

We estimate $\left(L_{1} \varphi_{j} \cdot \varphi_{l}\right)_{\rho_{0}}$ by splitting the integration over the regions $D_{a}$ and $D_{a}^{\prime}$. As before the integration over $D_{a}^{\prime}$ gives terms of order $\varepsilon(a) \lambda$ where $\varepsilon(a) \rightarrow 0$ as $a \rightarrow 1$. For the other terms

$$
\int_{D_{a}} \rho_{0} \eta_{k} \varphi_{j} \bar{\varphi}_{l} d x=\int_{D} \rho_{0} \eta_{k} \varphi_{J} \bar{\varphi}_{l} d x+\varepsilon(a) \lambda \equiv\left(\eta_{k} \varphi_{J} \cdot \varphi_{l}\right)_{\rho_{0}}+\varepsilon(a) \lambda .
$$


Now a straightforward calculation shows that $\left(\eta_{k} \varphi_{j} \cdot \varphi_{i}\right)_{\rho_{0}}=0$ except for the following.

$$
\begin{gathered}
\left(\eta_{1} \varphi_{2} \cdot \varphi_{3}\right)_{\rho_{0}}=\left(\eta_{1} \varphi_{3} \cdot \varphi_{2}\right)_{\rho_{0}}=[3 p \mu / 2]+o(\mu) . \\
\left(\eta_{2} \varphi_{2} \cdot \varphi_{3}\right)_{\rho_{0}}, \quad\left(\eta_{2} \varphi_{3} \cdot \varphi_{2}\right)_{\rho_{0}}, \quad\left(\eta_{2} \varphi_{4} \cdot \varphi_{k}\right)_{\rho_{0}} \in O\left(\lambda^{2}\right) .
\end{gathered}
$$

The terms $\left(R \varphi_{j} \cdot \varphi_{i}\right)_{\rho_{0}}$ used in calculating $\left(L_{1} \varphi_{j} \cdot \varphi_{i}\right)_{\rho_{0}}$ give contributions of order $O\left(\lambda^{2} \ln (1 / \lambda)\right)$ if $2 \leq i, j \leq 3, O\left(\lambda^{3 / 2}\right)$ if $i=2,3, j=4$ or $i=4, j=2,3, O(\lambda)$ if $i=j=4$. The remaining part of the operator $K_{1, u_{0}}^{\prime}$ cancels with the operator $K_{0}$ and contributes terms of order $\varepsilon(a) \lambda$ to the expressions $\left(L \varphi_{j} \cdot \varphi_{i}\right)_{\rho_{0}}$.

Finally we must show that $\left\|K_{1, u_{0}}^{\prime}-K_{0}\right\| \in O(\lambda \ln (1 / \lambda))$ which in turn will imply that $\left\|L_{1}\right\|$ and hence $\|L\| \in O(\lambda \ln (1 / \lambda))$. Using (4.47) we find that $\left\|K_{1, u_{0}}^{\prime}-K_{0}\right\|$ is bounded by

$$
\sup _{z_{0} \in D} \int_{D_{a}} k\left(z, z_{0}\right) \rho_{0}(z)[|\eta|+|R|] d x+\int_{D_{a}^{\prime}} k\left(z, z_{0}\right)\left[\lambda\left|f^{\prime}\right|^{2} e^{u_{0}}-\rho_{0}\right] d x .
$$

With the aid of the "special calculation" above it is easily checked that the upper bound on the first integral is $O[\lambda \ln (1 / \lambda)]$ while the second term is $O(\lambda)$.

Therefore $\|L\| \in O[\lambda \ln (1 / \lambda)]$ and since $M=(1-N)^{-1} L$ we have $\|M\| \in O[\lambda \ln (1 / \lambda)]$ as well. Now the eigenfunctions $\varphi_{t}=1,2,3,4$ are uniformly bounded in $\lambda$ as $\lambda \rightarrow 0$. Thus

$$
\left|\left(M^{2}(I-M)^{-1} \varphi_{j} \cdot \varphi_{i}\right)_{\rho_{0}}\right| \leq c \lambda^{2} \ln ^{2}(1 / \lambda)(1 \cdot 1)_{\rho_{0}} .
$$

But $(1 \cdot 1)_{\rho_{0}}=16 \pi /(1+\mu) \in O(1)$ as $\lambda \rightarrow 0$ and so

$$
\left(M^{2}(I-M)^{-1} \varphi_{i} \cdot \varphi_{J}\right)_{\rho_{0}} \in O\left[\lambda^{2} \ln ^{2}(1 / \lambda)\right] .
$$

Collecting the various terms that occur in (4.44) we find

$$
\begin{aligned}
& a_{22}=-3 \mu+o(\mu)+\varepsilon(a) \mu \\
& a_{33}=-3 \mu+o(\mu)+\varepsilon(a) \mu \\
& a_{23}=a_{32}=(3 p \mu) / 2+o(\mu)+\varepsilon(a) \mu \\
& a_{24}=a_{42}=O\left(\lambda^{3 / 2}\right)+\varepsilon(a) \mu \\
& a_{44}=-\frac{3}{2}[\ln (1 / \mu)]^{-1}+o\left[\ln ^{-1}(1 / \mu)\right]+\varepsilon(a) \mu .
\end{aligned}
$$

Now $p=f^{\prime \prime \prime}(0)$ satisfies $|p|<2$ and remains bounded away from \pm 2 for rectangles $\Omega(\alpha)$ as long as $\alpha$ remains bounded away from 0 or $\pi / 2$. For each of the terms in (4.48) we have limit $\varepsilon(a) \rightarrow 0$ as $a \rightarrow 1$. It follows that the matrix $\left(a_{i j}\right)$ is dominated by its leading terms and upon solving (4.43) we find that $\left|c_{t}\right| \leq r(\lambda)\|w\|$ where $r(\lambda) \in O(1 / \lambda)$. From our earlier discussion we may conclude that $\left\|\left(I-K_{u_{0}}^{\prime}\right)^{-1}\right\| \in O(1 / \lambda)$. The proof of Lemma 4.2 is complete. 
The necessary estimates have been made and we are now ready to apply the modified Newton iteration on the operator $K(u)$. We set

$$
\begin{aligned}
S(u) & =u-\left(I-K_{u_{0}}^{\prime}\right)^{-1}(u-K(u)) \\
& =\left(I-K_{u_{0}}^{\prime}\right)^{-1}\left(K(u)-K_{u_{0}}^{\prime}(u)\right)
\end{aligned}
$$

and will make use of the following result to be found in Vainberg [16, p. 260].

Convergence Theorem. Let $X$ be a Banach space and let $S(x)$ be a $C^{1}$-function from an open ball $B\left(x_{0}, R\right)=\left\{x \mid\left\|x-x_{0}\right\|<R\right\}$ into $X$. Suppose that there is a differentiable real-valued function $\varphi(t)$ defined on the interval $\left[0, t^{\prime}\right]$ where $t^{\prime}<R$ satisfying

(a) $\left\|S\left(x_{0}\right)-x_{0}\right\| \leq \varphi(0)$

(b) $\left\|S^{\prime}(x)\right\| \leq \varphi^{\prime}(t) \leq$ if $\left\|x-x_{0}\right\| \leq t$.

Suppose further that the equation $\varphi(t)=t$ has a root in $\left[0, t^{\prime}\right]$. If $\left\{x_{n}\right\}$ is the sequence with $x_{n+1}=S\left(x_{n}\right)$, then $x_{n} \rightarrow x^{*}$ where $S\left(x^{*}\right)=x^{*}$ and $\left\|x^{*}-x_{0}\right\| \leq t^{*}$ where $t^{*}$ is the smallest root of $\varphi(t)=t$ in $\left[0, t^{\prime}\right]$.

Suppose that we also have $\varphi\left(t^{\prime}\right) \leq t^{\prime}$. If $\varphi(t)=t$ has a unique solution $t^{*}$ in $\left[0, t^{\prime}\right]$, then the solution $x^{*}$ is unique in $B\left(x_{0}, t^{\prime}\right)=\Omega_{0}$ and successive approximations starting from any $x_{0}^{\prime} \in \Omega_{0}$ will converge to $x^{*}$.

We continue with the proof of Theorem 4.1. From (4.49) we have $S^{\prime}(u)(h)=\left(I-K_{u_{0}}^{\prime}\right)^{-1}\left(K_{u}^{\prime}-K_{u_{0}}^{\prime}\right)(h)$. We compute

$$
\begin{aligned}
K_{u}^{\prime}(h)-K_{u_{0}}^{\prime}(h) & =\left[K_{1, u}^{\prime}(h)-K_{1, u_{0}}^{\prime}(h)\right]+\left[K_{2, u}^{\prime}(h)-K_{2, u_{0}}^{\prime}(h)\right] \\
& =K_{1, u_{0}}^{\prime}\left[\left(e^{u-u_{0}}-1\right) h\right]+K_{2, u_{0}}^{\prime}\left[\left(e^{-\left(u-u_{0}\right)}-1\right) h\right] .
\end{aligned}
$$

Therefore, we find

$$
\left\|S_{u}^{\prime}\right\| \leq\left\|\left(I-K_{u_{0}}^{\prime}\right)^{-1}\right\|\left[\left\|K_{1, u_{0}}^{\prime}\right\|+\left\|K_{2, u_{0}}^{\prime}\right\|\right]\left(e^{t}-1\right)
$$

if $\left\|u-u_{0}\right\| \leq t$. But from Lemma 4.2 , we know that $\left\|\left(I-K_{u_{0}}^{\prime}\right)^{-1}\right\| \in$ $O(1 / \lambda)$ and $\left\|K_{1, u_{0}}^{\prime}\right\|,\left\|K_{2, u_{0}}^{\prime}\right\| \in O[\ln (1 / \lambda)]$. Therefore

$$
\left\|S_{u}^{\prime}\right\| \leq c[(1 / \lambda) \ln (1 / \lambda)]\left(e^{t}-1\right) \equiv \Gamma \cdot\left(e^{t}-1\right) \equiv \varphi^{\prime}(t)
$$

when $\left\|u-u_{0}\right\| \leq t$, where $c$ is some constant and $\Gamma=c[(1 / \lambda) \ln (1 / \lambda)]$. We now have

$$
\varphi(t)=\varphi(0)+\Gamma\left(e^{t}-1-t\right) .
$$

The equation $\varphi(t)=t$ will have exactly one root $t^{\prime}$ if $1+\varphi(0)=$ $(1+\Gamma) \ln [(1+\Gamma) / \Gamma]$ where $t^{\prime}=\ln [(1+\Gamma) / \Gamma]$. If $1+\varphi(0)<$ $(1+\Gamma) \ln [(1+\Gamma) / \Gamma], \varphi(0)>0$, then the equation $\varphi(t)=t$ will have exactly one root $t^{*}$ in the interval $\left[0, t^{\prime}\right]$. 
We now estimate $\left\|S\left(u_{0}\right)-u_{0}\right\|$.

$$
S\left(u_{0}\right)-u_{0}=-\left(I-K_{u_{0}}^{\prime}\right)^{-1}\left(u_{0}-K\left(u_{0}\right)\right) .
$$

From the results of Lemma 4.2 we find

$$
\left\|S\left(u_{0}\right)-u_{0}\right\| \leq b(1 / \lambda)\left[\lambda^{3} \ln (1 / \lambda)\right]=b \lambda^{2} \ln (1 / \lambda) \equiv \varphi(0)>0 .
$$

It is easy to check that $\varphi(0) /[(1+\Gamma) \ln [(1+\Gamma) / \Gamma]-1]$ has a limit of 0 as $\lambda \rightarrow 0$. Therefore the convergence theorem applies for $\lambda$ suitably small, ( $\lambda<\tilde{\lambda}$ say). Therefore, for $0<\lambda<\tilde{\lambda}$ there is a unique solution $u^{*}$ to $S\left(u^{*}\right)=u^{*}$ in $\overline{B\left(u_{0}, t^{\prime}\right)}$ so that

$$
\left\|u^{*}-u_{0}\right\| \leq t^{\prime}=\ln [(1+\Gamma) / \Gamma] \cong 1 / \Gamma=c \lambda / \ln (1 / \lambda) \rightarrow 0 \text { as } \lambda \rightarrow 0 \text {. }
$$

This shows in particular that $u^{*}(x, \lambda)$ also converges uniformly to $4 \ln (1 /|z|)$ on any subset of $\bar{D}$ which omits a neighborhood of the origin.

Finally, we note that our construction of the approximate solution $u_{0}(x, \alpha, \lambda)$ depends continuously on $\lambda, \alpha$. Each step in our construction of $u_{0}(x, \alpha, \lambda)$ is an explicit recipe each of which depends continuously on $\lambda$ and $\alpha$. Finally, the Newton iteration formula (4.49) is an operator depending continuously on $\alpha$ and $\lambda$. It follows that the mapping $(\alpha, \lambda) \rightarrow$ $u^{*}(x, \alpha, \lambda) \in C^{0}(\bar{D})$ is also continuous. Theorem 4.1 is proven.

We have constructed our desired family of solutions to the system $(3,3),(4,4)$ by mapping the rectangular domains $\Omega(\alpha)$ onto the unit disk and doing the analysis there. We now wish to consider the smooth dependence of these solutions on the parameters $\lambda$ and $\alpha$. This is most easily done by mapping a typical rectangular domain which we may choose to be of the form $[0,1] \times[0, \sqrt{b}] \equiv \Omega_{b}$ onto the unit square $\Omega_{1}=[0,1] \times[0,1]$ by a simple stretching and studying the transformed family on this fixed domain.

If $u_{x x}+u_{y y}+(\lambda / \sqrt{b}) f(u)=0$ on $\Omega_{b}$ with zero boundary values, then the transformed function will satisfy the P.D. E.

$$
u_{x x}+b^{-1} u_{y y}+(\lambda / \sqrt{b}) f(u)=0 \text { on } \Omega_{1} .
$$

This function will satisfy the corresponding integral equation

$$
\begin{aligned}
& u\left(\mathbf{x}_{0}\right)=\int_{\Omega_{1}} k\left(\mathbf{x}, \mathbf{x}_{0}, b\right)(\lambda f[u(\mathbf{x})]) d x \\
& k\left(\mathbf{x}, \mathbf{x}_{0}, b\right)=-(4 \pi)^{-1} \ln \left[\left(x-x_{0}\right)^{2}+b\left(y-y_{0}\right)^{2}\right] \\
&+h\left(\mathbf{x}, \mathbf{x}_{0}, b\right) .
\end{aligned}
$$

Here once again we are labeling a typical point in $\Omega_{1}$ by $\mathbf{x}=(x, y)$. The function $h\left(\mathbf{x}, \mathbf{x}_{0}, b\right)$ is " $b$-harmonic" on $\Omega_{1}$ satisfying the P.D.E. $h_{x x}+$ $b^{-1} h_{y y}=0$ on $\Omega_{1}$ and is chosen so that $k\left(\mathbf{x}, \mathbf{x}_{0}, b\right)$ vanishes for $\mathbf{x} \in \partial \Omega_{1}$. 
LEMMA 4.3. Consider the operator

$$
v\left(\mathbf{x}_{0}, b\right)=\int_{\Omega_{1}} k\left(\mathbf{x}, \mathbf{x}_{0}, b\right) u(\mathbf{x}) d \mathbf{x} \equiv T[b, u]\left(\mathbf{x}_{0}\right)
$$

The following are true.

(a) The mapping $T:(0, \infty) \times C\left(\bar{\Omega}_{1}\right) \rightarrow C\left(\bar{\Omega}_{1}\right)$, which is linear in the second variable, is a continuously differentiable map such that the image function vanishes on the boundary.

(b) The map $(b, u(\mathbf{x})) \rightarrow D_{l} v(\mathbf{x}, b)$ where $D_{l} v$ is a partial derivative with respect to one of the $\mathbf{x}$-coordinates, is a continuous map into the space $C\left(\Omega^{\prime}\right)$ where $\Omega^{\prime}$ is any domain with $\bar{\Omega}^{\prime} \subset \Omega_{1}$. The mapping $(b, u(\mathbf{x})) \rightarrow D_{i} v_{b}$ is also a continuous map into $C\left(\Omega^{\prime}\right)$.

Proof. We split the operator $T[b, u]$ by setting

$$
\begin{aligned}
v(\mathbf{x}, b) & =T[b, u](\mathbf{x})=w(\mathbf{x}, b)+\chi(\mathbf{x}, b) \\
w\left(\mathbf{x}_{0}, b\right) & =-\int_{\Omega_{1}}(4 \pi)^{-1} \ln \left[\left(x-x_{0}\right)^{2}+b\left(y-y_{0}\right)^{2}\right] u(\mathbf{x}) d \mathbf{x} \\
\chi\left(\mathbf{x}_{0}, b\right) & =\int_{\Omega_{1}} h\left(\mathbf{x}, \mathbf{x}_{0}, b\right) u(\mathbf{x}) d \mathbf{x} .
\end{aligned}
$$

Using standard methods from potential theory (e.g. Gilbarg and Trudinger [7, p. 53]) it is seen that $w(\mathbf{x}, b), w_{b}(\mathbf{x}, b), D_{\imath} w(\mathbf{x}, b), D_{\imath} w_{b}(\mathbf{x}, b)$ are all continuous on $\bar{\Omega}_{1}$ and are calculated by differentiating under the integral sign. (The kernels for $w, w_{b}$ are homogeneous of degree 0 while the remaining kernels are of degree -1$)$. Furthermore the mappings $(b, u(\mathbf{x}))$ $\rightarrow\left(w, w_{b}, D_{i} w, D_{i} w_{b}\right)$ are all continuous from $(0, \infty) \times C\left(\bar{\Omega}_{1}\right) \rightarrow C\left(\bar{\Omega}_{1}\right)$.

Now $\chi(\mathbf{x}, b) \in C\left(\bar{\Omega}_{1}\right) \cap C^{\infty}\left(\Omega_{1}\right)$ is a $b$-harmonic function on $\Omega_{1}$ with $\chi=-\omega$ on $\partial \Omega_{1}$. It follows that the mappings $(b, u(\mathbf{x})) \rightarrow\left[\chi(\mathbf{x}, b), \chi_{b}(\mathbf{x}, v)\right]$ are continuous maps from $(0, \infty) \times C\left(\bar{\Omega}_{1}\right) \rightarrow C\left(\bar{\Omega}_{1}\right)$ while the derivative maps $D_{i} \chi(\mathbf{x}, b), D_{i} \chi_{b}(\mathbf{x}, b)$ are continuous maps into the space $C\left(\Omega^{\prime}\right)$ where $\bar{\Omega}^{\prime} \subset \Omega_{1}$.

It follows from our discussion that the map $T:(0, \infty) \times C\left(\bar{\Omega}_{1}\right) \rightarrow$ $C\left(\bar{\Omega}_{1}\right)$ is continuous. Since $T$ is linear in $u$ we have for the derivative of $T$

$$
D T[b, u](\Delta b, h)=v_{b}(\mathbf{x}, b) \Delta b+T[b, h]
$$

for $\Delta b \in R$ and $h(\mathbf{x}) \in C\left(\bar{\Omega}_{1}\right)$. From this we see that $D T$ is also continuous and so $T$ is of class $C^{1}$.

LEMMA 4.4. Let $(\alpha, \lambda) \rightarrow u^{*}(\mathbf{x}, \alpha, \lambda)$ be the continuous mapping into $C(\bar{D})$ constructed in Theorem 4.1 where $u^{*}-K\left(u^{*}\right)=0$, and $K(u)$ is the integral operator (4.4). The derivative map $\left(I-K_{u^{*}}^{\prime}\right): C(\bar{D}) \rightarrow C(\bar{D})$ 
is invertible for each $u^{*}(\mathbf{x}, \alpha, \lambda)$ with $\lambda>0$ and satisfies the bound $\left\|\left(I-K_{u^{*}}^{\prime}\right)^{-1}\right\| \in O(1 / \lambda)$

Proof. By Lemma 4.2 we have $\left\|\left(I-K_{u_{0}}^{\prime}\right)^{-1}\right\| \in O(1 / \lambda)$ where $u_{0}$ was the initial approximate solution. Now

$$
\begin{aligned}
\left(I-K_{u}^{\prime}\right) & =\left(I-K_{u_{0}}^{\prime}\right)\left[I-\left(I-K_{u_{0}}^{\prime}\right)^{-1}\left(K_{u}^{\prime}-K_{u_{0}}^{\prime}\right)\right] \\
& =\left(I-K_{u_{0}}^{\prime}\right)\left(I-S_{u}^{\prime}\right)
\end{aligned}
$$

where $S(u)$ is the operator (4.49) used in the modified Newton procedure. It was shown that $\left\|S_{u}^{\prime}\right\| \leq \Gamma\left(e^{t}-1\right)$ if $\left\|u-u_{0}\right\| \leq t$ where $\Gamma=c[(1 / \lambda) \ln (1 / \lambda)]$ for some constant $c$. However, $\left\|u^{*}-u_{0}\right\|<t^{*}$ where $t^{*} \in\left[0, t^{\prime}\right]$ is the smallest positive root of $t=\varphi(t)=\varphi(0)+\Gamma\left(e^{t}-1-t\right)$ where $\varphi(0) \leq b \lambda^{2} \ln (1 / \lambda)$ as $\lambda \rightarrow 0$. It is easy to check that $\varphi(0) / t^{*} \rightarrow 1$ as $\lambda \rightarrow 0$, hence $t^{*}(\lambda) \in O\left[\lambda^{2} \ln (1 / \lambda)\right]$. From this we obtain $\left\|S_{u}^{\prime}\right\| \in$ $O\left[\lambda \ln ^{2}(1 / \lambda)\right]$. Therefore $\left\|S_{u^{*}}^{\prime}\right\| \rightarrow 0$ as $\lambda \rightarrow 0$ and from the identity above we conclude that $\left(I-K_{u^{*}}^{\prime}\right)$ is invertible and $\left\|\left(I-K_{u^{*}}^{\prime}\right)^{-1}\right\| \in O(1 / \lambda)$.

Now take the functions $u^{*}(\mathbf{x}, \alpha, \lambda)$ constructed in Theorem 4.1 on the disk domain and transform them to our new standard domain $\Omega_{1}$, the unit square. For convenience we replace the shape factor $\alpha$ by the parameter $b$. The transformed function $u(\mathbf{x}, b, \lambda), \mathbf{x} \in \bar{\Omega}_{1}$, is then a continuous mapping $(b, \lambda) \rightarrow u(\mathbf{x}, b, \lambda) \in C\left(\bar{\Omega}_{1}\right)$ which vanishes on the boundary and is a solution to the operator equation

$$
u-2 \lambda T[b, \sinh u]=0
$$

where $T(b, u)$ is the integral operator defined in (4.51), (4.52).

THEOREM 4.2. The continuous family $(b, \lambda) \rightarrow u(\mathbf{x}, b, \lambda)$ is a $C^{2}$-mapping from the admissible set $0 \subset R^{2} \rightarrow C\left(\bar{\Omega}_{1}\right)$. The following are also true.

(a) $u(\mathbf{x}, b, \lambda) \in C^{1}\left(\Omega^{\prime}\right)$ for any $\bar{\Omega}^{\prime} \subset \Omega_{1}$.

(b) $u(\mathbf{x}, b, \lambda) \in C^{\infty}\left(\Omega_{1}\right) \cap C^{0}\left(\bar{\Omega}_{1}\right)$ for each $(b, \lambda)$ and is a solution to $(4.50)$.

(c) The mappings $(b, \lambda) \rightarrow\left[u(\mathbf{x}, b, \lambda), u_{b}(\mathbf{x}, b, \lambda), u_{\lambda}(\mathbf{x}, b, \lambda)\right]$ are continuous into $C\left(\bar{\Omega}_{1}\right)$ while the maps $(b, \lambda) \rightarrow$ $\left[D_{i} u(\mathbf{x}, b, \lambda), D_{i} u_{b}(\mathbf{x}, b, \lambda)\right]$ are continuous into $C\left(\Omega^{\prime}\right)$ where $\bar{\Omega}^{\prime} \subset \Omega_{1}$.

Proof. From Theorem 4.1 we have that $u(\mathbf{x}, b, \lambda)$ depends continuously on $(b, \lambda)$, and by the discussion above is a solution to the operator equation (4.53) for $\lambda>0, F(u, b, \lambda)=u-2 \lambda T[b, \sinh u]=0$. From 
Lemma 4.3 it follows that $F(u, b, \lambda)$ is a $C^{1}$-function from $R^{2} \times C\left(\bar{\Omega}_{1}\right)$ $\rightarrow C\left(\bar{\Omega}_{1}\right)$. Furthermore, the derivative

$$
F_{u}(u, b, \lambda)(h)=I(h)-2 \lambda T[b,(\cosh u)](h)
$$

is invertible for small positive $\lambda$ by Lemma 4.4. Therefore by the Implicit Function Theorem $u(\mathbf{x}, b, \lambda)$ depends differentiably on $(b, \lambda)$ as a mapping $(b, \lambda) \in 0 \subset R^{2} \rightarrow C\left(\bar{\Omega}_{1}\right)$.

Now since $u=2 \lambda T[b, \sinh u]$ and since $\sinh u$ is continuous on $\bar{\Omega}_{1}$, it follows from Lemma 4.3 that $u \in C^{1}\left(\Omega^{\prime}\right)$ for $\bar{\Omega}^{\prime} \subset \Omega_{1}$. But then it follows that $u \in C^{\infty}\left(\Omega^{\prime}\right)$ and is a solution to the D.E. (4.50) on $\Omega_{1}$. The remaining smoothness properties also follow from Lemma 4.3.

LemmA 4.5. Let $u(x, y) \in C^{0}(\bar{\Omega}) \cap C^{2}(\Omega)$ be a solution to the D.E. $\Delta u+F(u)=0$ on $\Omega$ where $\Omega$ is a rectangular domain $(0, A) \times(0, B)$ and $F(u)$ is an odd $(F(-u)=-F(u))$ continuously differentiable function with $F(u)$ positive for positive $u$, and let $u=0$ on $\partial \Omega$. Let $\tilde{u}(x, y)$ be the extension of $u(x, y)$ to all of $R^{2}$ obtained by odd reflection of $u(x, y)$ across the rectangular grid determined by $\Omega$. Then $u(x, y) \in C^{2}\left(R^{2}\right)$ and is $a$ solution to $\Delta u+F(u)=0$ on $R^{2}$.

Proof. This result must be well known. The fact that reflection across a line is smooth is proven in the same manner as the case of harmonic functions [2, p. 272]. The resulting extension is then continuous on all of $R^{2}$ and is a smooth solution to the D.E. except possibly at the vertices. From this it follows easily that the extension is in fact smooth across the vertices as well.

One may conclude that the family $u(\mathbf{x}, b, \lambda)$ satisfying (4.53) on $\bar{\Omega}_{1}$ and discussed in Theorem 4.2 has an odd $C^{\infty}$ (analytic, in fact) extension to all of $R^{2}$, being a solution to the D. E. (4.50) and vanishing on the grid lines $x=k, y=l$ ( $k, l$ integers). We now show that these extensions depend continuously and differentiably on $(b, \lambda)$.

THEOREM 4.3. Let $\tilde{u}(\mathbf{x}, b, \lambda)$ be the odd smooth extension of the family $u(\mathbf{x}, b, \lambda)$ described in Theorem 4.2 The following are true. On any compact domain $\Omega \subset R^{2}$ the following functions are continuous maps from the parameter set $(b, \lambda) \in O \subset R^{2} \rightarrow C(\bar{\Omega})$ where $\lambda$ is positive.

(a) $(b, \lambda) \rightarrow \tilde{u}(\mathbf{x}, b, \lambda)$

(b) $(b, \lambda) \rightarrow D_{l} \tilde{u}(\mathbf{x}, b, \lambda)$

(c) $(b, \lambda) \rightarrow \tilde{u}_{\lambda}(\mathbf{x}, b, \lambda), \tilde{u}_{b}(\mathbf{x}, b, \lambda)$

(d) $(b, \lambda) \rightarrow D_{i} \tilde{u}_{b}(\mathbf{x}, b, \lambda)$. 
Proof. Let $\Omega_{2}=[-1,1] \times[-1,1]$ be the square of edge 2 . On $\bar{\Omega}_{2}$, the map $(b, \lambda) \rightarrow \tilde{u}(\mathbf{x}, b, \lambda) \in C\left(\bar{\Omega}_{2}\right)$ is continuous (for $\left.\lambda>0\right)$ and is a solution to the integral equation (4.53) where now the kernel function $k\left(\mathbf{x}, \mathbf{x}_{0}, b\right)$ for the operator $T$ is relative to $\Omega_{2}$ rather than $\Omega_{1}$. The conclusions of Theorem 4.2 can now be applied to this enlarged domain. So for any subdomain $\bar{\Omega}^{\prime} \subset \Omega_{2}$ the functions listed above are continuous mappings into $C\left(\bar{\Omega}^{\prime}\right)$. Since the functions are periodic, the result follows.

We now shall discuss the behavior of the family of functions $u(\mathbf{x}, b, \lambda)$ described in Theorems 4.1-4.3 as the parameter $\lambda \rightarrow 0$. We suppose that the functions have been extended as described in Lemma 4.5 so that the domain includes all $\mathrm{x} \in R^{2}$. From Theorem 4.1 we know that if $\Omega \subset R^{2}$ is any domain whose closure avoids the center of any square in the grid determined by $\Omega_{1}$ and if the shape parameter $b$ remains inside some compact subinterval of $(0, \infty)$ then as $\lambda \rightarrow 0$ the function $u(\mathbf{x}, b, \lambda)$ converges uniformly to the function $u_{0}(\mathbf{x}, b)$ where for $\mathbf{x} \in \Omega_{1}$

$$
u_{0}(\mathbf{x}, b)=4 \ln (1 /|g(\mathbf{x}, b)|) \text {. }
$$

Here $g(\mathbf{x}, b)$ is the nonconformal map of $\Omega_{1}$ onto the unit disk $D$ obtained by first squashing $\Omega_{1}$ onto the rectangle $\Omega_{b}=[0,1] \times[0, \sqrt{b}]$ and then conformally mapping $\Omega_{b}$ onto $D . u_{0}(\mathbf{x}, b)$ is then extended oddly to all $\mathbf{x} \in R^{2}$ as described in Lemma 4.5. This limit function $u_{0}(\mathbf{x}, b)$ is " $b$ harmonic" [satisfies $u_{x x}+b^{-1} u_{y y}=0$ ].

THEOREM 4.4. Let $\Omega \subset R^{2}$ be any domain whose closure excludes the centers of any square in the grid formed by $\Omega_{1}$, and suppose $b$ is constrained to remain in some compact subinterval of $(0, \infty)$. Then as $\lambda \rightarrow 0$, $D^{k} u(\mathbf{x}, b, \lambda) \rightarrow D^{k} u_{0}(\mathbf{x}, b, \lambda)$ where $D^{k} u$ is any partial derivative of $u(\mathbf{x}, b, \lambda)$ with respect to the $x$-coordinates.

Proof. We may suppose that $\Omega$ is a disk domain whose closure does not contain the center of any square. On $\Omega$ we have

$$
\begin{aligned}
& u_{x x}+b^{-1} u_{y y}+(\lambda / \sqrt{b})\left(e^{u}-e^{-u}\right)=0 \text { on } \Omega \\
& u(\mathbf{x}, b, \lambda)=\Psi(\mathbf{x}, b, \lambda) \text { on } \partial \Omega .
\end{aligned}
$$

As an integral equation we may write

$$
\begin{aligned}
u\left(\mathbf{x}_{0}, b, \lambda\right) & =\int_{\Omega} k\left(\mathbf{x}, \mathbf{x}_{0}, b\right)\left[\lambda\left(e^{u}-e^{-u}\right)\right] d \mathbf{x}+h\left(\mathbf{x}_{0}, b, \lambda\right) \\
& =v\left(\mathbf{x}_{0}, b, \lambda\right)+h\left(\mathbf{x}_{0}, b, \lambda\right)
\end{aligned}
$$


Here $k\left(\mathbf{x}, \mathbf{x}_{0}, b\right)$ is the Green's kernel (4.51) adjusted to the disk $\Omega$ and $h(\mathbf{x}, b, \lambda)$ is a $b$-harmonic function with $h=\Psi$ on $\partial \Omega$. Since $u(\mathbf{x}, b, \lambda) \rightarrow$ $u_{0}(\mathbf{x}, b)$ uniformly on $\Omega$ as $\lambda \rightarrow 0$ it is clear that (4.55) remains valid down to the limit, $\lambda=0$. But now using the arguments in the proofs of Lemma 4.3 and Theorem 3.2 we can conclude that on any subdomain $\bar{\Omega}^{\prime} \subset \Omega$ the functions $u, u_{x}, u_{y}$ converge to $u_{0},\left(u_{0}\right)_{x}$ and $\left(u_{0}\right)_{y}$ as $\lambda \rightarrow 0$. This process can be iterated and we can conclude that $D^{k} u(\mathbf{x}, b, \lambda) \rightarrow D^{k} u_{0}(\mathbf{x}, b)$ as $\lambda \rightarrow 0$ and $\mathbf{x} \in \Omega^{\prime}$.

The following lemma concerns the symmetry properties of the solutions $u(\mathbf{x}, b, \lambda)$.

LEMMA 4.6. Let $\Omega$ be the rectangle $[-a, a] \times[-b, b] \subset R^{2}$ centered about the origin. Let $F(u)$ be an odd function of class $C^{1}$ which is positive for positive $u$, and suppose $u(x, y) \in C^{2}(\Omega)$ is a solution to $u_{x x}+u_{y y}+F(u)$ $=0$ which is positive inside $\Omega$ and equals zero on the boundary. The following are true:

(a) $u(x, y)=u(-x, y)=u(x,-y)$ so that $u(x, y)$ is an even function of $(x, y)$. Hence $u_{x}(x, y)$ is odd in $x$, even in $y$ (e.t.c.).

(b) For a fixed $y, 0 \leq y<b, u(x, y)$ is a strictly decreasing function of $x$ for $0 \leq x \leq a$. Similarly for a fixed $x, 0 \leq x<a u(x, y)$ is strictly decreasing in $y, 0 \leq y \leq b$.

(c) $u_{y}(x, b)$ is strictly increasing for $0 \leq x \leq a$ from $u_{y}(0, b)<0$ to $u_{y}(a, b)=0$.

Proof. We first observe that by the E. Hopf boundary point principle, along the edges of $\Omega$ the outward normal derivative $\partial u / \partial \nu<0$, and by the boundary point principle at a corner due to J. Serrin [14], $u_{x y}$ does not vanish at any corner. These observations allow us to apply the passing planes technique initially due to A. D. Alexandrov and extended in the paper of Gidas-Ni-Nirenberg [6], (see also [17]).

We reflect $u(x, y)$ about lines parallel to the coordinate axes and apply the Hopf touching principle or the Hopf boundary point touching principle (see [17] for details). The conclusions are

(a) $u(x, y)$ is an even function in $x$ and $y$.

(b) $u(x, y)$ for a fixed $y,-b<y<b$, strictly increases from $0=$ $u(-a, y)$ to a maximum at $u(0, y)$ and then strictly decreases back to $0=u(a, y)$. A similar result holds in the $y$-direction.

(c) $u_{y}(x, b)$ strictly increases on $[0, a]$ from $u_{y}(0, b)<0$ to $u_{y}(a, b)$ $=0$. A similar result holds for $u_{x}(a, y), 0 \leq y \leq b$. 
The symmetry properties of $u(\mathbf{x}, b, \lambda)$ as described in Lemma 4.6 actually follow from our method of construction. We observe that the functions $u(\mathbf{x}, b, \lambda)$ constructed in Theorem 4.1 are not guaranteed to be positive on the interior of the domain. However, we have the following result.

Lemma 4.7. The functions $u(\mathbf{x}, b, \lambda)$ described in Theorem 4.2-4.3 are positive on $\Omega_{1}$.

Proof. Suppose $u(\mathbf{x}, b, \lambda)$ is defined for $(b, \lambda) \in 0 \subset R^{2}$ where 0 is a connected set. If we can show that $u(\mathbf{x}, b, \lambda)$ is positive on $\Omega_{1}$ for one $(\tilde{b}, \tilde{\lambda}) \in 0$, then $u(\mathbf{x}, b, \lambda)$ will be positive on $\Omega_{1}$ for all $(b, \lambda) \in 0$. This is a direct consequence of the E. Hopf boundary point principle and Serrin's boundary point principle at a corner, discussed in the proof of the previous lemma.

By Theorem 4.3 we have $u(\mathbf{x}, b, \lambda) \Rightarrow u_{0}(\mathbf{x}, b)$ uniformly on any subdomain $\Omega^{\prime}$ of $\Omega_{1}$ which does not contain the center of $\Omega_{1}$ as $\lambda \rightarrow 0$. Here $u_{0}(\mathbf{x}, b)$ is given by (4.54). Therefore, if $\Omega_{0}$ is any subdomain of $\Omega_{1}$ with $\bar{\Omega}_{0} \subset \Omega_{1}$, then fixing $b=\tilde{b}$, there exists $\tilde{\lambda}>0$ so that for $0<\lambda<\tilde{\lambda}$ we have $u(\mathbf{x}, \tilde{b}, \lambda) \geq \varepsilon>0$ for $\mathbf{x} \in \Omega_{0}$.

Now let $p$ be a boundary point of $\Omega_{1}$ which is not a corner. In a neighborhood of $p$, the inward normal $\partial u_{0} / \partial v$ is positive. But $\partial u / \partial v \Rightarrow$ $\partial u_{0} / \partial \nu$ uniformly on this neighborhood by Theorem 4.4 . It follows that for $\lambda$ sufficiently small $u(\mathbf{x}, \tilde{b}, \lambda)$ will be positive for $\mathbf{x} \in U_{p} \cap \Omega_{1}$ where $U_{p}$ is a neighborhood of $p$.

Finally consider a corner. Here we have $\left(u_{0}\right)_{x y} \neq 0$ and the same will be true for $u_{x y}$ for $\lambda$ sufficiently small, $b=\tilde{b}$, and $\mathbf{x}$ in some neighborhood of the corner. It now follows that there is a $\lambda$ so that, for $0<\lambda<\tilde{\lambda}$, $u(\mathbf{x}, \tilde{b}, \lambda)$ will be positive for $\mathbf{x} \in U \cap \Omega_{1}$ where $U$ is some neighborhood of the corner. But $\bar{\Omega}_{1}$ is compact and the Lemma follows.

V. Conclusion with pictures. Let $\Omega=(0, A) \times(0, B)$ be a rectangle chosen so that the smallest eigenvalue of the system $\Delta v+\gamma v=0$ on $\Omega, v \mid \partial \Omega=0$ is $\gamma_{1}=1$. In particular we have $\gamma_{1}=1=\pi^{2}\left[1 / A^{2}+1 / B^{2}\right]$ and so we see that both $A$ and $B$ are greater than $\pi$. If one solves the P.D.E.

$$
\Delta W+2 \lambda \sinh W \cosh W=0 \quad \text { on } \Omega, \quad W=0 \quad \text { on } \partial \Omega
$$

or equivalently setting $2 W=\Sigma$ the system (3.3) $\Delta \Sigma+2 \lambda \sinh \Sigma=0$, then by shrinking the domain we have a function $\omega(u, v)$ defined on $\sqrt{2 \lambda} \Omega \equiv$ $\Omega(\lambda)$ and satisfying

(3.5) $\Delta \omega+\sinh \omega \cosh \omega=0 \quad$ on $\Omega(\lambda), \quad \omega=0 \quad$ on $\partial \Omega(\lambda)$. 
From here we follow the procedure of $\S \S I I$ and III to construct an immersed surface $\mathbf{x}(u, v, \lambda)$ with fundamental domain $\Omega(\lambda)$. In our pictures we shall choose $H=1 / 2$.

As $2 \lambda \rightarrow \gamma_{1}=1$ (i.e. $\lambda \rightarrow 1 / 2$ ) the functions $W(u, v, \lambda) \Rightarrow 0$ uniformly. There is a bifurcation from the trivial solution $W \equiv 0$ to (5.1) when $\lambda=1 / 2$. The discussion of $\S I V$ established a one-parameter family $W(u, v, \lambda)$ depending smoothly on $\lambda$ near $\lambda=0(\lambda>0)$ such that $W(u, v, \lambda)$ converges to $W_{0}(u, v)=2 \ln (1 /|g(w, \Omega)|)$ where $g(w, \Omega)$ is the inverse of the Schwartz-Christoffel mapping from the unit disk to the rectangle $\Omega$.

The following statements concerning solutions to the system (5.1) are worth bearing in mind.

LEMMA 5.1. Let $W$ be any positive solution to the system (5.1). It follows that $0<2 \lambda<\gamma_{1}$ where $\gamma_{1}$ is the smallest eigenvalue to the Laplace equation with zero boundary data.

Proof. Essentially well known. Follows from the fact that $\sinh W>W$ for $W$ positive.

LeMma 5.2. For every $\lambda, 0<2 \lambda<\gamma_{1}$ there exists a positive solution to (5.1).

Proof. Not to be given here. Uses so-called mountain pass lemma.

If one imagines the solution curve $[W(u, v, \lambda), \lambda]$ in the " $(W, \lambda)$ plane", then one is tempted to believe that the curve bifurcating from $\lambda=1 / 2$ connects smoothly to the large family near $\lambda=0$. This has not yet been established. The following conjecture is especially tempting.

Conjecture. Let $W\left(u, v, \lambda_{1}\right)$ and $W\left(u, v, \lambda_{2}\right)$ be two positive solutions to (5.1) where $\Omega$ is a rectangular domain. If $\lambda_{1}<\lambda_{2}$ then $W\left(u, v, \lambda_{1}\right)>$ $W\left(u, v, \lambda_{2}\right)$ for each point $(u, v) \in \Omega$.

Remark. In the case of a disk domain this conjecture is true.

Now suppose we have our positive solution $\omega(u, v)$ to (3.5) defined on $\Omega(\lambda)$ and then extended as a solution to all of $R^{2}$ from which we construct our surface $\mathbf{x}(u, v, \lambda)$ with mean curvature $H=1 / 2$. As $2 \lambda \rightarrow 1$ we know that $\omega \rightarrow 0$. In this limit case $\mathbf{x}(u, v, \lambda)$ (with $\lambda=1 / 2$ ) is a representation of a pure cylinder whose cross-section is a circle of radius one. We shall sketch the image of the rectangle indicated as [1-6] in the 
illustrations. So if the fundamental rectangle is $\Omega(\lambda)=(0, A) \times(0, B)$ where $A=\sqrt{2 \lambda} A_{0}, B=\sqrt{2 \lambda} B_{0}$ then we sketch the image of the rectangular region whose vertices are $(A / 2,0),(A / 2, B / 2),(3 A / 2,0)$, $(3 A / 2, B / 2)$. The rest of the immersion can then be obtained by a rotation of $180^{\circ}$ of the pictured region about the normal line through the image of $(2)=\mathbf{x}(A, 0)$ and then subsequent reflections.

We have sketched four figures starting with $\lambda=1 / 2$ (the cylinder) and then surfaces corresponding to decreasing values of $\lambda$ until the planes $\Pi_{0}$ and $\Pi_{1}$ become identical so that the immersed surface closes up in one direction at least. The following observations are useful to keep in mind as $\lambda \rightarrow 0$. We let $\Omega^{+}(\lambda)$ denote a rectangular domain where $\omega(u, v)$ is positive (e.g. $\Omega^{+}(\lambda)=\Omega(\lambda)$ ). In this domain the principle curvatures $k_{1}$, $k_{2}$ are both positive, hence the Gauss curvature $K$ is positive and the surface $\mathbf{x}\left(\Omega^{+}\right)$is convex. $\Omega^{-}(\lambda)$ is any congruent rectangle where $\omega(u, v)$ is negative. In this case $k_{1}<0$ and $k_{2}>0$ so that Gauss curvature is negative.

THEOREM 5.1. As $\lambda \rightarrow 0$ the following are true.

(a) The area of the image of the Gauss map

$$
=\int_{\mathbf{x}\left(\Omega^{+}(\lambda)\right)} K d A \text { approaches } 4 \pi
$$

(b) The area of the image surface, $\mathbf{x}\left[\Omega^{+}(\lambda)\right]$ approaches $4 \pi(2)^{2}=16 \pi$.

(c) The area of the image surface, $\mathbf{x}\left[\Omega^{-}(\lambda)\right]$ approaches zero.

Proof. The proofs of the assertions are similar and direct. For (a) we have

$$
\begin{aligned}
\iint_{x\left[\Omega^{+}(\lambda)\right]} K d A & =\iint_{\Omega^{+}(\lambda)} \sinh \omega \cosh \omega d u d v \\
& =\iint_{\Omega^{+}} \sinh W \cosh W(2 \lambda) d u d v \\
& =-\iint_{\Omega^{+}} \Delta W d u d v \\
& =-\int_{\partial \Omega^{+}} \frac{\partial W}{\partial \nu} d s \rightarrow-\int_{\partial \Omega^{+}} \frac{\partial W_{0}}{\partial \nu} d s \\
& =-2 \int_{\partial D} \frac{\partial \ln (1 / r)}{\partial \nu} d s=4 \pi .
\end{aligned}
$$


A similar calculation for (b) gives for the area of the image $\mathbf{x}\left[\Omega^{+}(\lambda)\right]$ the formula

$$
\text { Area }=2 \lambda \iint_{\Omega^{+}} e^{-\Sigma} d u d v-2 \iint_{\Omega^{+}} \Delta \Sigma d u d v
$$

As $\lambda$ approaches 0 the first term in easily seen to approach zero and the whole expression converges to $16 \pi$. The proof of (c) is even more direct.

Our calculations suggest that the surface $\mathbf{x}\left(\Omega^{+}(\lambda)\right)$ converges towards a sphere of radius 2. The surface $\mathbf{x}\left(\Omega^{-}(\lambda)\right)$ has negative Gauss curvature (and small area) and serves to connect the sphere-like pieces together.

Finally we note that as $\lambda \rightarrow 0$ the fundamental rectangle $\Omega(\lambda)$ shrinks to a point. If $H=1 / 2$, then along the line $v=0$ we have $\omega(u, 0)=0$ and $E=\left|\mathbf{x}_{u}\right|^{2}=1$. This shows that $\mathbf{x}(u, 0)$ is parameterized by arc length. Thus the curve $\mathbf{x}(u, 0), 0 \leq u \leq A(\lambda)=\sqrt{2 \lambda} A_{0}$ has length $\sqrt{2 \lambda} A_{0}$ which shrinks to zero. Similar remarks apply to the image of any of the grid lines $u=m A, v=n B$ along which $\omega=0$.

Figure 1: The Schwartz-Christoffel Map (3.2)

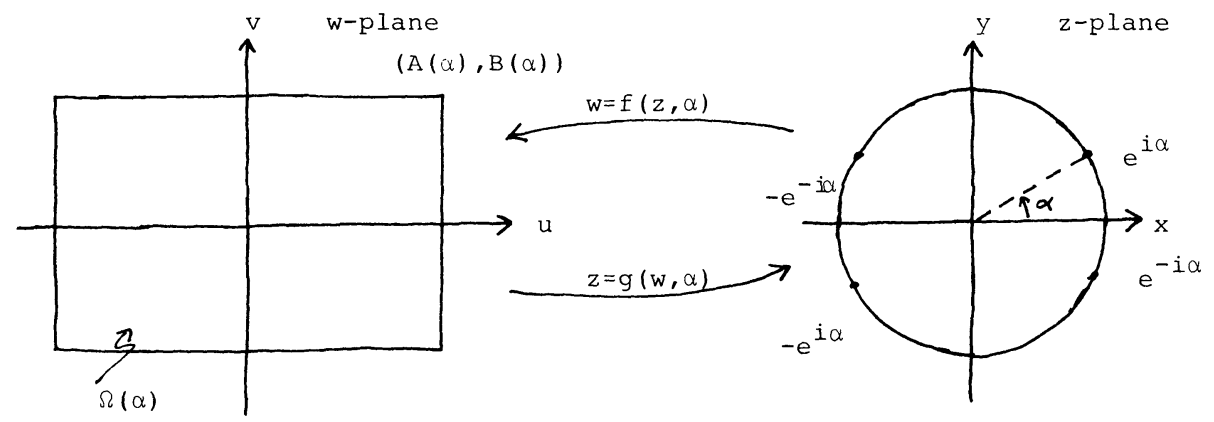

FIGURE 2. The Fundamental Domain $\Omega(\alpha, \lambda)=\sqrt{2 \lambda} \Omega(\alpha)$
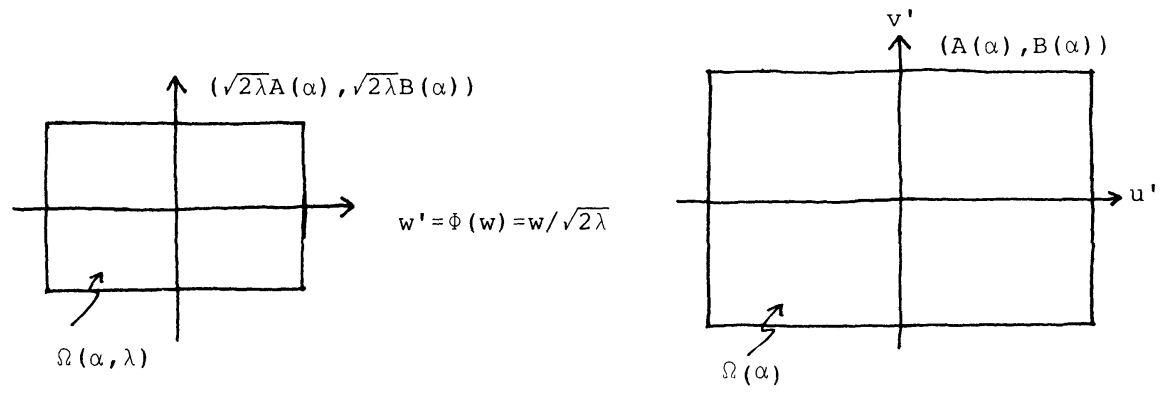

1. $\Delta \Psi+\lambda\left|f^{\prime}(z, \alpha)\right|^{2}\left(e^{\Psi}-e^{-\Psi}\right)=0$ on $D, \Psi \mid \partial D=0$ $\operatorname{limit} \Psi(z, \alpha, \lambda)=4 \ln (1 /|z|)$ as $\lambda \rightarrow 0$ 
2. $\Delta \Sigma+\lambda\left(e^{\Sigma}-e^{-\Sigma}\right)=\Delta \Sigma+2 \lambda \sinh \Sigma=0$ on $\Omega(\alpha)$ where $\Sigma=\Psi \circ g$

3. $\Delta W+2 \lambda \sinh W \cosh W=0$ where $2 W=\Sigma$

4. $\Delta \sigma+\sinh \sigma=0$ on $\Omega(\alpha, \lambda)$ where $\sigma=\Sigma \circ \Phi$

5. $\Delta \omega+\sinh \omega \cosh \omega=0$ where $2 \omega=\sigma$

6. $x(u, v, \alpha, \lambda)$ is solution to (2.19) with fundamental domain $\Omega(\alpha, \lambda)$ while $y\left(u^{\prime}, v^{\prime}, \alpha, \lambda\right)=\left[x \circ \Phi^{-1}\left(u^{\prime}, v^{\prime}, \alpha, \lambda\right)\right] / \sqrt{2 \lambda}$ is a solution to (3.7) with fundamental domain $\Omega(\alpha)$.

FIGURE 3: Graph of $\mathbf{y}(u,-B(\alpha), \alpha, \lambda)$.
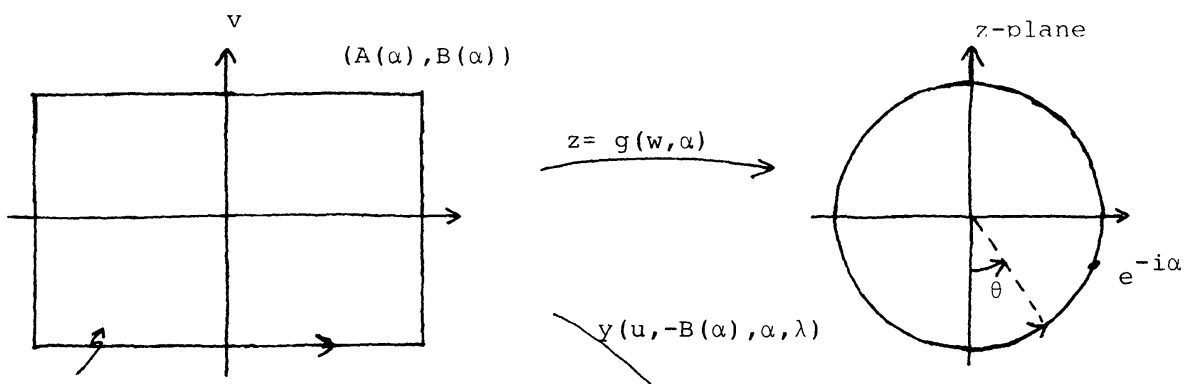

$\Omega(\alpha)$

i) $\mathbf{y}(u+4 A(\alpha), v, \alpha, \lambda)$

$=\mathbf{y}(u, v, \alpha, \lambda)+4 S(\alpha, \lambda) \mathbf{e}$

ii) $\mathbf{y}(u, v, \alpha, \lambda) \rightarrow \mathbf{y}_{0}(u, v)$

as $\lambda$ approaches 0 .

iii) When $\lambda$ equals 0

then $\phi$ equals $2 \theta$.

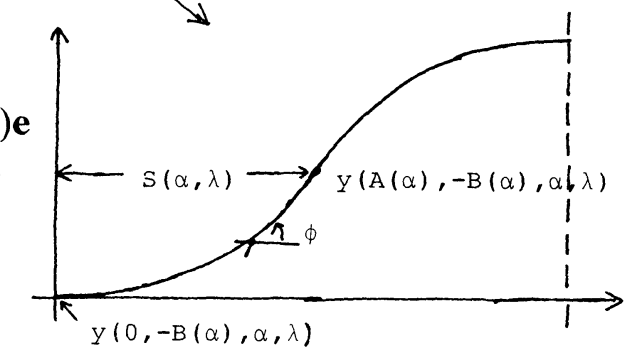

FIgURE 4: Graphs of $C_{\alpha}=\mathbf{y}_{0}(u,-B(\alpha))$
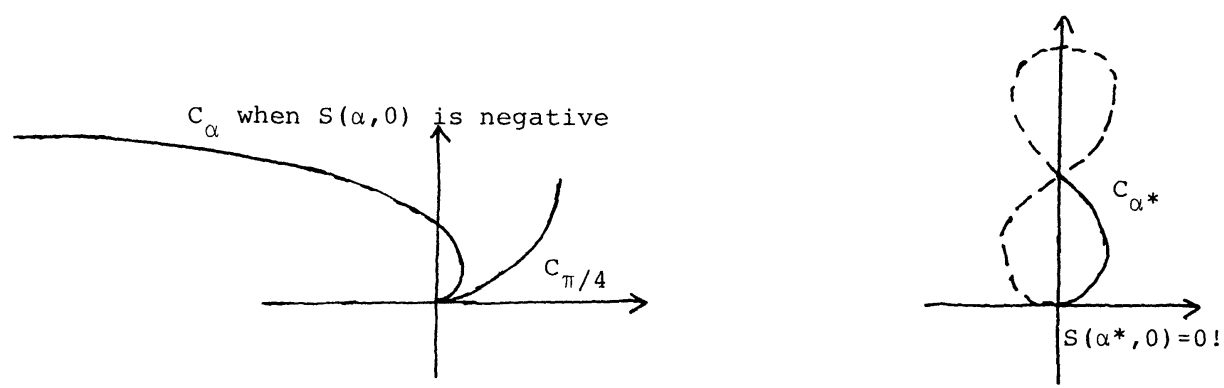
Figure 5: Suggestive Illustrations.

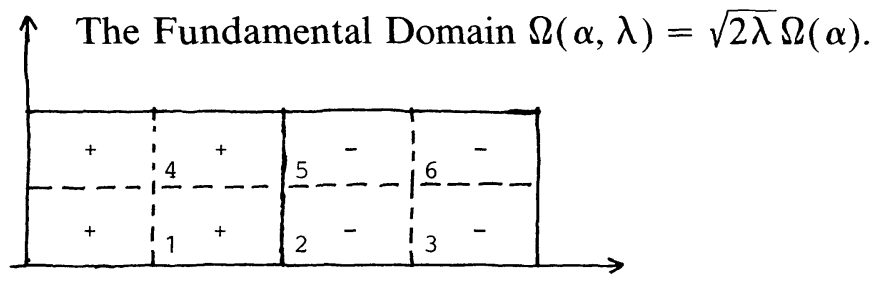

Case 1: $(W=0, \lambda=1 / 2)$ The pure cylinder

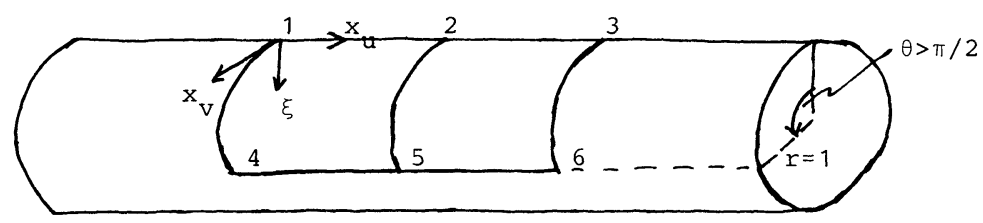

Case 2: ( $W$ positive but not too large, $\lambda$ less than $1 / 2)$

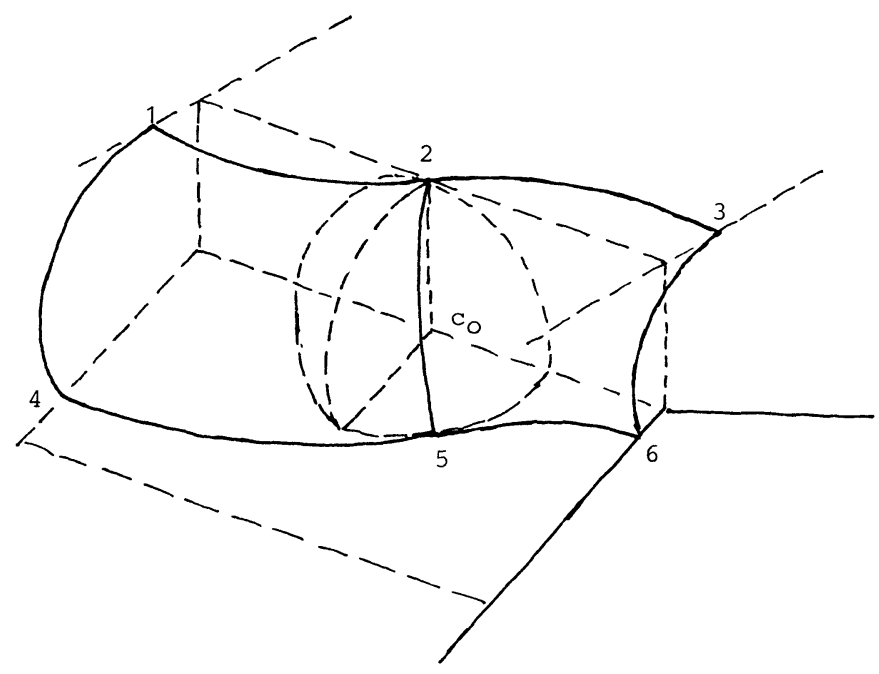


Case 3: ( $W$ larger, but the planes $\Pi_{0}, \Pi_{1}$ still separated)

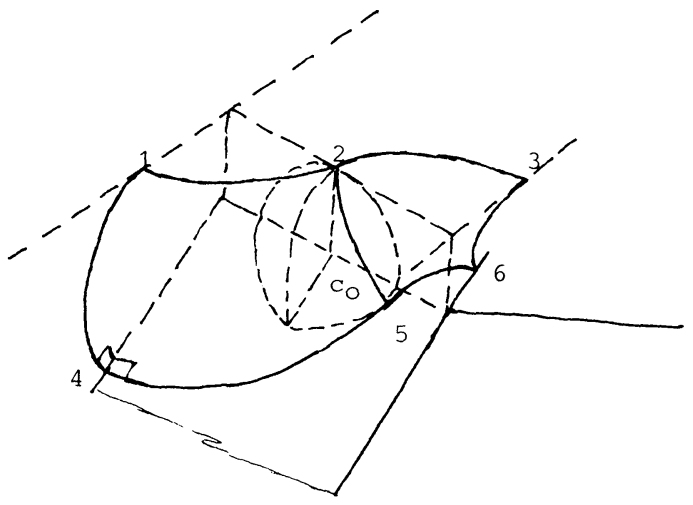

Case 4: (The planes $\Pi_{0}$ and $\Pi_{1}$ are identical)

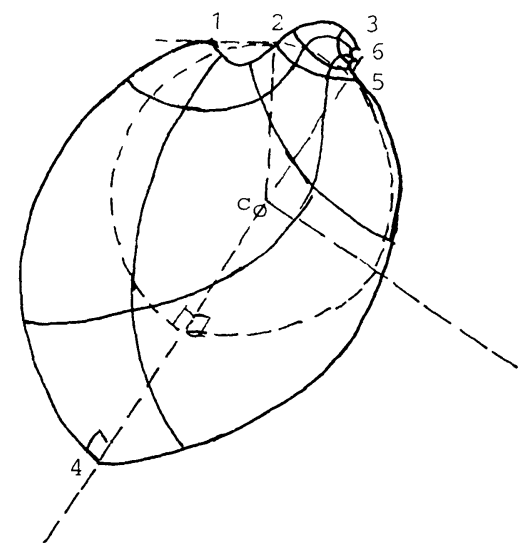

Final Note: If one reflects the sketched figure about the plane of the paper $\Pi_{0}$, then a surface in the shape of a "clam shell" is obtained. Now rotate this figure about a vertical line through $\mathbf{c}_{0}$. The completed figure resembles a clam with the shells opened up a bit.

\section{REFERENCES}

[1] A. D. Alexandrov, Uniqueness theorems for surfaces in the large, V. Vestnik, Leningrad Univ. 13, No. 19 (1958), 5-8. Amer. Math. Soc. Trans. (Series 2) 21, 412-416.

[2] R. Courant and D. Hilbert, Methods of Mathematical Physics, Vol. II, Wiley Interscience, (1962).

[3] M. P. do Carmo, Differential Geometry of Curves and surfaces, Prentice Hall, (1976).

[4] L. P. Eisenhart, A Treatise on the Differential Geometry of Curves and Surfaces, Dover Reprint (1960).

[5] P. R. Garabedian, Partial Differential Equations, John Wiley \& Sons, (1964). 
[6] B. Gidas, W. Ni and L. Nirenberg, Symmetry and related properties via the maximum principle, Comm. Math. Physics, 68 No. 3 (1979), 209-343.

[7] D. Gilbarg and N. S. Trudinger, Elliptic Partial Differential Equations of Second Order, 2nd Edition, Springer Verlag, (1983).

[8] H. Hopf, Differential Geometry in the Large (Seminar Lectures New York University 1946 and Stanford University 1956), Lecture Notes in Mathematics 1000, Springer Verlag (1983).

[9] Wu-Yi Hsiang, Generalized rotational hypersurfaces of constant mean curvature in the Euclidean spaces, I, J. Differential Geometry, 17 (1982), 337-356.

[10] J.H. Jellett, Sur la Surface dont la Courbure Moyenne est Constante, J. Math. Pures Appl., 18 (1853), 163-167.

[11] N. N. Lebedev, Special Functions and their Applications, Dover (1972).

[12] J. L. Moseley, A two-dimensional Dirichlet problem with an exponential nonlinearity, SIAM J. Math. Anal., 14, No. 5 (1983), 934-946.

[13] J. L. Moseley, On Asymptotic Solutions for a Dirichlet Problem with an Exponential Nonlinearity, REP AMR I, West Virginia Univ., (1981).

[14] J. Serrin, A symmetry problem in potential theory, Arch. Rational Mech. Anal., 43 (1971), 304-318.

[15] J. J. Stoker, Differential Geometry, Wiley Interscience (1969).

[16] M. M. Vainberg, Variational Methods for the Study of Nonlinear Operators, HoldenDay Inc. (1964).

[17] H. C. Wente, The Symmetry of Sessile and Pendent Drops, Pacific J. Math., 88, No. 2 (1980), 387-397.

[18] V. H. Weston, On the Asymptotic Solution of a Partial Differential Equation with an Exponential Nonlinarity, SIAM J. Math. Anal., 9 (1978), 1030-1053.

Received August 8, 1984. The author gratefully acknowledges the support of the MaxPlanck-Institute für Mathematik, Bonn, W. Germany.

THE UNIVERSITY OF TOLEDO

TOLEDO, OH 43606 



\section{PACIFIC JOURNAL OF MATHEMATICS EDITORS}

V. S. VARADARAJAN (Managing Editor)
University of California
Los Angeles, CA 90024
HEBERT CLEMENS
University of Utah
Salt Lake City, UT 84112
CHARLES R. DEPRIMA
California Institute of Technology
Pasadena, CA 91125

\section{R. FINN}

Stanford University

Stanford, CA 94305

HeRmanN FlaschKa

University of Arizona

Tucson, AZ 85721

RAMESH A. GANGOLli

University of Washington

Seattle, WA 98195

ROBION KIRBY

University of California

Berkeley, CA 94720

\section{C. MoOre}

University of California

Berkeley, CA 94720

H. SAMELSON

Stanford University

Stanford, CA 94305

HAROLD STARK

University of California, San Diego

La Jolla, CA 92093

ASSOCIATE EDITORS
R. ARENS
E. F. BECKENBACH
B. H. NeUmanN
F. WOLF
K. Yoshida (1906-1982)

\section{SUPPORTING INSTITUTIONS}

UNIVERSITY OF ARIZONA

UNIVERSITY OF BRITISH COLUMBIA

CALIFORNIA INSTITUTE OF TECHNOLOGY

UNIVERSITY OF CALIFORNIA

MONTANA STATE UNIVERSITY

UNIVERSITY OF NEVADA, RENO

NEW MEXICO STATE UNIVERSITY

OREGON STATE UNIVERSITY
UNIVERSITY OF OREGON

UNIVERSITY OF SOUTHERN CALIFORNIA

STANFORD UNIVERSITY

UNIVERSITY OF HAWAII

UNIVERSITY OF TOKYO

UNIVERSITY OF UTAH

WASHINGTON STATE UNIVERSITY

UNIVERSITY OF WASHINGTON 


\section{Pacific Journal of Mathematics}

\section{Vol. 121, No. $1 \quad$ November, 1986}

Om P. Agrawal, Douglas Napier Clark and Ronald George Douglas,

Invariant subspaces in the polydisk $\ldots \ldots \ldots \ldots \ldots \ldots \ldots \ldots \ldots \ldots$

Christoph Bandt and Gebreselassie Baraki, Metrically invariant measures

on locally homogeneous spaces and hyperspaces $\ldots \ldots \ldots \ldots \ldots \ldots \ldots$

Marcy Mason Barge, Horseshoe maps and inverse limits ..............29

Russell Gene Bilyeu, Robert Richard Kallman and Paul Weldon Lewis,

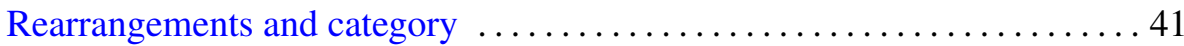

Jean Bourgain, A problem of Douglas and Rudin on factorization . . .......47

Hernan Cendra, A normal form and integration in finite terms for a class of

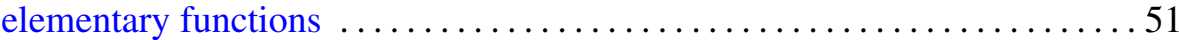

Ky Fan, The angular derivative of an operator-valued analytic function . . . . 67

Gerhard Gierz, On the Dunford-Pettis property of function modules of

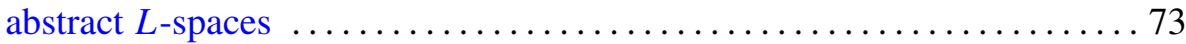

Gabriel Katz, On polynomial generators in the algebra of complex functions

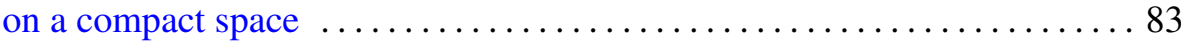

Ridgley Lange, Duality and asymptotic spectral decompositions $\ldots \ldots \ldots . .93$

Anthony To-Ming Lau and Peter F. Mah, Quasinormal structures for certain spaces of operators on a Hilbert space ................... 109

R. Daniel Mauldin, Correction: "The set of continuous nowhere differentiable functions"

Alan Harvey Mekler and Saharon Shelah, $\omega$-elongations and Crawley's problem

Alan Harvey Mekler and Saharon Shelah, The solution to Crawley's problem

Richard Rochberg, Deformation of uniform algebras on Riemann surfaces

Joseph Roitberg, On weak epimorphisms in homotopy theory

Jesús M. Ruiz, A remark on fields with the dense orbits property

Henry Wente, Counterexample to a conjecture of H. Hopf

David G. Wright, Rigid sets in $E^{n}$ 